\title{
Fluorescent multiplex cell rolling assay: simultaneous capturing up to seven samples in real-time using spectral confocal microscopy
}

\author{
Ayman F. AbuElela, Asma S. Al-Amoodi, Amal J. Ali and Jasmeen S. Merzaban* \\ Cell Migration and Signaling Laboratory, King Abdullah University of Science and Technology, \\ Biological and Environmental Sciences and Engineering Division, Thuwal, Saudi Arabia
}

\section{* Correspondence:}

Jasmeen S. Merzaban

4700 King Abdullah University of Science and Technology

Thuwal, Saudi Arabia

23955-6900

jasmeen.merzaban@kaust.edu.sa

Keywords: deglycosylation, selectins, migration, sialylation, glycans, live cell imaging, multicolor fluorescence tracking, parallel plate flow chamber assay 


\section{ABSTRACT}

The parallel plate flow chamber assay is widely utilized to study physiological cell-cell adhesive interactions under dynamic flow that mimics the blood stream. In this technique, the cells are perfused under defined shear stresses over a monolayer of endothelial cells (expressing homing molecules eg. selectins) or a surface (expressing recombinant homing molecules). However, with the need to study multiple samples and multiple parameters per sample, using a traditional bright-field microscopebased-flow-assay allows only one sample at a time to be analyzed resulting in: high inter-experiment variability, the need for normalization, waste of materials and significant consumption of time. We developed a multiplexing approach using a three-color fluorescence staining method, which allowed for up to seven different combination signatures to be run at one time. Using this Fluorescent Multiplex Cell Rolling-(FMCR) assay each sample is labeled with a different signature of emission wavelengths and mixed with other samples just minutes before the flow run. Subsequently, real-time images are acquired in a single pass using a line-scanning spectral confocal microscope. To illustrate the glycan-dependent binding of E-selectin, a central molecule in cell migration, to its glycosylated ligands expressed on myeloid-leukemic cells in flow, the FMCR assay was used to analyze Eselectin-Ligand interactions following the addition (fucosyltransferase-treatment) or removal (deglycosylation) of key glycans on the flowing cells. The FMCR assay allowed us to analyze the cell-adhesion events from these different treatment conditions simultaneously in a competitive manner and to calculate differences in rolling frequency, velocity and tethering capability of cells under study. 


\section{INTRODUCTION}

Cell migration relies on the adhesion interaction between surface proteins of the circulating cells (eg. selectin ligands) and their counter receptors on the vascular endothelium (eg. E/P-selectin). ${ }^{1-9}$ Such processes can be probed in vitro using cell adhesion assays ${ }^{10-13}$ which are adaptable tools that can be used to understand proliferation and cellular adhesion in the context of inflammation and cancer cell metastasis. ${ }^{14-17}$ Moreover, they can also be used to discover novel drugs that could inhibit ${ }^{18-20}$ or enhance ${ }^{21}$ adhesion in disease settings or to understand the dynamics of drug/nanoparticle absorption by endothelial cells under physiologic flow ${ }^{22,23}$ among other uses.

To date, the analysis of rolling capabilities of cells has been largely studied in vitro using parallel plate flow chamber (PPFC)-based assays. ${ }^{14-16,24-34}$ These assays mimic cells migrating through the circulation where flowing cells are infused under shear stress over a monolayer of a substrateexpressing cells (or a Polyvinylidene Difluoride (PVDF) membrane boasting immunoprecipitated ligands of interest adsorbed on its surface) seeded on the surface of one of the parallel plates of the setup. $^{14,15,24,28-30,32,33}$ Using a bright-field microscope and video recording system to image the process, each sample or cell type is analyzed in a singleplex mode as it is perfused over a monolayer of substrate-expressing cells (or a substrate-coated surface) under defined shear stresses controlled by a syringe pump. Although a powerful tool, its design has a number of inherent technical limitations including inter-experimental variations stemming from pipetting errors and cell count errors that result in variations in the numbers of substrate-expressing cells in a given plate (seeding variation) and differences in the expression of substrates among cells (substrate expression disparity). Additionally, separation of control and test samples and the absence of intra-experiment replication lead to the need for normalization. As a low-throughput technique, PPFC is time-consuming and relatively tedious. Moreover, the potential for unintentional experimental bias is high since the researcher usually chooses the field of view to analyze, which may influence the reproducibility due to surface variation. 
Although a few trials have described methods to overcome some of these deficiencies, ${ }^{16,27}$ they make use of customized dual camera emission splitting platforms that would allow for capturing two samples simultaneously in real-time and solves the inter-experimental variation problems, it is lowthroughput. ${ }^{16}$

Fluorescent cell barcoding ${ }^{35,36}$ and multiplexing approaches to simultaneously measure different cell types that have unique fluorescence identifiers (UFIs) has been used in a number of imaging techniques, including a study that imaged seven different cell types in fixed tissue samples. ${ }^{34}$ The approaches alluded to in these studies require that the cells or tissues first be fixed likely due to the nature of coupling the dyes/fluorophores used. With the fluorescent barcoding approach, for example, cells are often fixed and permeabilized to allow the amine-reactive dyes to access protein targets efficiently which results in a higher intensity staining. ${ }^{35-37}$ In addition, the barcoding approach requires that consideration be taken to the concentration of dye and the methanol content of the buffer being used for the staining, which should be optimized for each cell type/experiment. Most importantly, multiplexed/multicolor experiments should be carefully compensated to avoid emission's bleedthrough of the various dyes into neighboring channels. This demands the development of fluorophores with minimal spectral overlap and experimental design that deals with the large number of fluorophores and their spectral overlap. Here, we introduce a relatively simpler multiplexing approach and apply it to the conventional cell flow assay, that we refer to as the Florescence Multiplex Cell Rolling (FMCR) assay, which uses a lower number of UFIs to label specific populations within a mixture of differentially and uniquely labeled cells (Figure 1A). To achieve this, we used a threecolor fluorescence staining approach that allows seven different combinations of UFIs to be run in a flow experiment at one time. Real-time images were then acquired in a single pass using a linescanning spectral confocal microscope. This technique allowed for the analysis of adhesion events from up to seven different treatment conditions simultaneously in real-time and for calculation of the 
differences in rolling frequency, velocity and tethering capability of cells under study (Figure 1B). This assay also permitted the analysis of rolling cell populations on an endothelial cell layer (or a selectin-coated surface) where populations are competing for occupancy of available substrate sites in a real-time scenario, a characteristic much like what occurs in an in vivo setting. The assay is easily adopted since it is performed using commercially available setups and software. 


\section{EXPERIMENTAL SECTION}

\section{Cells:}

The human cell line KG1a (human acute myelogenous leukemia) serves as hematopoietic stem/progenitor cell like $\left(\mathrm{CD} 34^{+}\right)$model cell; they were purchased from ATCC and cultured in RPMI 1640 medium supplemented with $10 \%$ FBS (Gibco) and 100 units/mL penicillin/streptomycin (Invitrogen). A transgenic Chinese hamster ovary (CHO) cell line stably expressing full-length mouse E-selectin (CHO-E) (or the plasmid alone (CHO-Mock) was established in our laboratory as described previously. ${ }^{38} \mathrm{CHO}$ cells were cultured in MEM medium containing 1x non-essential amino acids (Invitrogen), 1x sodium pyruvate (Invitrogen), 10\% $\quad$ FBS (Gibco) and 100 units $/ \mathrm{mL}$ penicillin/streptomycin (Invitrogen) and prepared for experiments as described under "Preparation of substrate-expressing cells for FMCR" in Methods of Supporting Information file. K562 (human Erythroleukemia cell line) cells were purchased from ATCC and cultured in IMDM medium with 10\% FBS (Gibco) and 100 units/mL penicillin/streptomycin (Invitrogen).

\section{Fluorescent multiplex cell rolling (FMCR):}

24-36 h prior to the cell flow experiment, sterile $40 \mathrm{~mm}$ coverslips (Fisher Scientific) in small plate were prepared with $1 \mathrm{X}$ attachment factor for $1 \mathrm{~h}$ prior to plating $2 \times 10^{6}$ E-selectin expressing $\mathrm{CHO}$ cells (CHO-E). The CHO-E cells were allowed to reach 90\% confluency. On the day of the experiment, KG-1a cells were stained with $5 \mu \mathrm{g} / \mathrm{mL}$ anti-CD34 (mouse $\mathrm{IgG}$, clone 8G12) primary antibody while $\mathrm{K} 562$ cells were stained with $5 \mu \mathrm{g} / \mathrm{mL}$ of anti-CD43 (mouse IgG, clone L60) primary antibody for 30 minutes at $4^{\circ} \mathrm{C}$ followed by washing with HBSS twice. Note that these antibodies do not block the interactions of ligands (i.e. CD34, CD44, PSGL-1 and CD43) to E-selectin as illustrated in a previous publication from our lab. ${ }^{38}$ To generate a unique fluorescent identifier (UFI) for each sample/treatment condition, anti-mouse IgG secondary antibodies conjugated with various fluorophores (AF405, AF546 and/or AF680) were used either alone or in combination (mixture of 2 
or 3 fluorophores) to stain the primary antibodies at a concentration of $5 \mu \mathrm{g} / \mathrm{mL}$ for $15-30$ minutes at $4^{\circ} \mathrm{C}$. Samples were then thoroughly washed at least 3 times with HBSS buffer. [Note: we used a combination of multiple fluorophores to produce a number of UFIs through the labeling of specific cell surface molecules. This could also be achieved using a variety of cell dyes or cell membrane permeable dyes that have minimal spectral overlap. There is a myriad of dyes available commercially from vendors such as Molecular Probes/ThermoFisher, but attention should be given to the intensity of the dyes used relative to one another and that the dyes do not emit wavelengths that overlap, which could require the use of proper/special lasers/filters and/or compensation. Our barcoding methods can also be integrated with existing methods ${ }^{35-37}$ to provide strategies to increase the multiplexity.]

Spectral overlap was ruled out using FACS analysis (see "Fluorescence Activated Cell Sorting (FACS)" in Methods of Supporting Information file) and confocal fluorescence imaging on single, double and triple stained samples with the proper adjustment of the laser power and optical gain using the AxioImager (Ziess). For the majority of experiments these settings were: power values (2-10\%), optical gain (adjusted according to signal intensity), pinhole width (maximum), frame size (512 x 512 pixels) and scanning rate (1 frame/sec) but adjustments were made depending on the staining signal intensity of the samples. Minutes prior to the introduction of the cells to the CHO-E monolayer, the UFI-labeled cell populations were accurately counted using Countess Automated Cell Counter (Invitrogen) and mixed at an equal ratio. A small sample of the mixed sample was then tested by the Countess Automated Cell Counter to ensure relatively equal ratios of cells were used using the appropriate EVOS light cubes (Invitrogen). The CHO-E cell coverslips were assembled in the FCS3 flow cell as per the manufacturer's instructions (BiopTech; https://bioptechs.com/product/fcs3$\underline{\text { starter-set/ }})$ where the inlet tube was placed into a buffer containing the cells under study ( $2 \mathrm{mM}$ $\mathrm{CaCl}_{2}$-since the binding of E-selectin to its ligands is $\mathrm{Ca}^{2+}$ dependent- in HBSS buffer at a concentration of $1-2 \times 10^{6}$ cells $/ \mathrm{mL}$ ) and the outlet tube was connected to the PHD 2000 syringe 
pump (Harvard Apparatus). When looking at selectin/selectin ligand interactions, the system was infused with ethylenediaminetetraacetic acid (EDTA) after the flow run to remove the specific $\mathrm{Ca}^{2+}$ dependent binding in order to detect non-specific binding events that will be subtracted during the analysis. Cell flow was controlled by a syringe pump that was programmed to start the flow at 0.2 dyne $/ \mathrm{cm}^{2}$ for 2 minutes and then the flow was increased at 30 -second intervals from 1 to 10 dynes $/ \mathrm{cm}^{2}$ (i.e. $1,2,3,4,5,7.5$ and $10 \mathrm{dyne} / \mathrm{cm}^{2}$ ). The syringe pump was programmed in a step-wise manner with different flow rates corresponding to pre-assigned shear stress values according to the following Equation.

$$
\tau=\frac{6 Q \mu}{b^{2}}
$$

where $\tau$ is the shear stress, $\mathrm{Q}$ is the flow rate, $\mu$ is the viscosity of the buffer, $\mathrm{b}$ is the flow chamber width and $\mathrm{h}$ is the height of the flow chamber. The experimental run started by running the syringe pump while imaging the cells in 3 detector channels simultaneously in time-lapse mode. Videos were then analyzed for event tracking using Imaris software (Bitplane). Data representation and statistical analysis was performed by a custom analysis script using R software (see "FMCR Sample Analysis Pipeline" in Supporting Information file).

\section{Cell tracking using the Imaris software:}

Cell tracking analysis began with intensity thresholding and smoothing. In order to use the spot detection algorithm, the intensity profile of each cell needed to be smoothed using the Gaussian blur filter in order to remove the empty pixels. Then, intensity profiles were inspected for each UFI to determine the intensity cut-off values at which the software can distinguish between the positive and negative signals at each channel. The next step was spot detection using the connected components algorithm. The algorithm was applied individually for each UFI using the previously detected intensity thresholds for profile capture. For example, the purple profile is a red + , blue + and green- 
where $(+)$ or $(-)$ can be determined by comparing the sum of the intensity profile of one spot to the threshold assigned at the previous step. At all the tested shear stress intervals, spot detection was followed by spot tracking using the auto regressive motion algorithm where the spots representing the same cell were connected to each other throughout the video to form a movement track. One track is the functional unit of the analysis of the rolling events as the rolling behavior parameters, are exported by track. Filtering of valid rolling tracks was performed next by removing the tracks representing nonspecific binding. Finally, the process was completed with exporting the tracking files for subsequent statistical analysis and data representation (see "Data representation and statistical analysis using the R software package" in Methods of Supporting Information file and "FMCR Sample Analysis Pipeline" in Supporting Information file).

\section{Hypothesis testing:}

Multiple hypothesis testing was performed using one-way analysis of variance (ANOVA) followed by multiple pairwise comparisons Tukey's honest significant difference (HSD) testing. Two-sample hypothesis testing was performed using Student's T-test.

\section{RESULTS AND DISCUSSION}

Adjustments were made to some of the elements of PPFC assay to achieve our objective of developing a simple, multiplex, and competitive in vitro migration assay. Namely, our methodology, the microscope setup, the preparation of the substrate-expressing cells (e.g. E-selectin-expressing Chinese hamster ovary $(\mathrm{CHO})$ cells) and the preparation of UFI-tagged cells. Moreover, we developed a comprehensive analysis pipeline using the R statistical analysis environment and Imaris software (see "FMCR Sample Analysis Pipeline" in Supporting Information file).

\section{The FMCR assay setup}


In the place of the conventional bright-field microscope used in the PPFC assay, ${ }^{14-16,24-33}$ the FMCR assay uses a confocal laser-scanning microscope (Figure S1) that allows the use of up to seven samples simultaneously through the combination of three fluorophores and the use of three detection filters. In addition, the materials required for this assay included (Figure S1): (i) a cell suspension of a mixture of cells (eg. KG-1a cells) tagged with UFIs (Figure 1A, Figure S2; see "Fluorescent multiplex cell rolling (FMCR)" in the Experimental Section); (ii) a monolayer of substrate-expressing cells (e.g. E-selectin-expressing CHO cells; see "Preparation of substrate expressing cells for FMCR" in Methods of the Supporting Information file); (iii) a parallel plate flow chamber with the lower plate (a cover slip) coated with a monolayer of the substrate-expressing cells and placed on the stage of an inverted microscope; (iv) an inverted microscope equipped with laser-scanning spectral confocal system; (v) a computer for time-lapse image capturing and storage; and (vi) a syringe pump for cell infusion under a defined step-wise program of shear stress magnitudes.

\section{Cell tagging with UFIs}

In order to prepare the distinct signatures needed for the rolling experiments, each cell specimen (e.g. cell type or treatment condition) was labeled with a UFI comprised of Alexa Fluor 405-, Alexa Fluor 546- or Alexa Fluor 680-conjugated antibodies or combinations thereof (see "Fluorescent multiplex cell rolling" in the Experimental Section). These tags were chosen to generate the minimum possible spectral overlap in order to allow distinguishable signals for each sample under simultaneous excitation and hence sufficiently short scanning time ( $\sim 900 \mathrm{~ms} /$ frame at $512 \times 512$ pixels). To describe the technology and investigate the potential bias the UFI may have on the detection of rolling cells in this setup, seven identical samples using an E-selectin-ligand-expressing cells (human myelogenous leukemia; KG-1a) were used (Figure 1A, Figure S2). Flow cytometric analysis of the fluorescence signal for each fluorescence tag showed insensible spectral overlap with other tags for any given UFI in non-compensated settings (Figure S2B). Before mixing the samples for the cell flow assay, the 
confocal acquisition parameters including laser power (to control for blue, green and red range staining intensity) and optical gain were adjusted so that no signal crosstalk was observed in any UFI in single-samples images (data not shown). A relevant study used Fourier spectroscopy and singular value decomposition to image seven cell types in a fixed tissue, each labeled with different fluorescence dyes. ${ }^{34}$ The assay described in this study, however, requires that the cells or tissues be fixed. Most importantly, since this assay requires multiple fluorophores to achieve multiplexing, the risk of bleed-through of these fluorophores to neighboring channels is much higher and therefore requires sophisticated mathematical models and analysis to deconvolute the emission signals. Our approach, on the other hand, allows for the use of live (unfixed) cells, minimizes the need for compensation if the fluorophores/dyes (up to 3) are chosen with little to no spectral overlap (Figure S2; note no compensation is needed for these fluorophores).

\section{The flow run and the time-lapse imaging}

Before running the flow experiment, the cell specimens under study (i.e. the KG-1a cells) were investigated individually for their unique tagging and spectral overlap (Figure S2). This step is critical to ensure faultless cell tracking during the video analysis. Proper laser power values (2-10\%), optical gain (adjusted according to signal intensity), pinhole width (maximum), frame size (512 x 512 pixels) and scanning rate (1 frame/sec) were adjusted as needed and depend on the staining signal intensity of the samples. Before assembling the PPFC device, running buffer containing $2 \mathrm{mM} \mathrm{CaCl}_{2}$ was infused into the flow chamber in order to remove all the air from the device. The syringe pump was set up with a step-wise program with increasing magnitudes of flow rates every 30 -second interval projecting the range of shear stress values studied (i.e. ranging from 2 dyne $/ \mathrm{cm}^{2}$ up to $20 \mathrm{dyne} / \mathrm{cm}^{2}$ ). At this point, the PPFC was assembled using a flow chamber kit from Bioptech (see "Fluorescent multiplex cell rolling" in the Experimental Section) where the cover slip with the substrate-expressing cells (i.e. CHO-E cells) was included (Figure S1). Differentially UFI-tagged KG-1a cells were 
counted and thoroughly and equally mixed at the required density (usually $10^{6}$ cells $/ \mathrm{mL}$ ) just prior to perfusion. The acquisition software was prepared for time-lapse mode with 1 frame/second for an amount of time exactly equal to the length of the syringe pump program (i.e. a step-wise program set to increase the flow rate every 30 -second interval starting at 2 dyne $/ \mathrm{cm}^{2}$ up to 20 dyne $/ \mathrm{cm}^{2}$ depending on the experiment). The UFI-tagged KG-1a cells were then perfused over the confluent monolayer of CHO-E cells at shear stresses programmed into the syringe pump. [Note: if the flow goes beyond 5 minutes, gentle pipetting of the UFI-tagged sample can be considered to ensure that mixing of cells is maintained throughout the experiment.] The software recorded videos of the flow run with the tethering and rolling events of interest. For controls, to detect non-specific binding events of E-selectin to its ligands, we infused the chamber with EDTA after the flow run to remove the specific $\mathrm{Ca}^{2+}$ dependent binding. ${ }^{38,39}$ This setup allows for imaging under dynamic flow conditions down to 900 $\mathrm{ms} /$ frame (at 512X512 pixels) and using the new Airyscanning system (Zeiss) would allow for even lower frame sizes. Interestingly, a high-throughput multi-well microfluidics system has been described $^{21,27}$ to study multiple samples individually in a multi-well setting. This setup has similar high-throughput capacity to ours except that it does not allow for samples to be run simultaneously under competitive settings on the same substrate expressing cells.

Once the flow run was complete, the raw files of the time-lapse experiment were transferred to Imaris software for cell tracking (Figure 1B; see "Cell tracking using Imaris software" in the Experimental Section). Our novel technique significantly increases the number of degrees of freedom from $<10$ to few hundreds, which brought about a significant increase in the statistical power of the assay by many orders of magnitude. For example, in the PPFC assay the number of events used to calculate the different rolling parameters are on the order tens or less ${ }^{17,} 19,38-41$ whereas in the FMCR assay described here, the numbers of events are in the range of hundreds and thousands.

\section{Detection bias}


Compared to the conventional cell rolling assay, the FMCR technique allows for minimal user curation and automated detection, and hence less time consumption and bias. To determine if the fluorescent identifier on KG-1a cells creates a detection bias in the rolling events detected, seven populations of KG-1a cells were prepared, each with a unique fluorescence identifier (Figure S2). KG-1a cells express E-selectin ligands and thus will interact and roll over E-selectin expressing CHO cells (CHO-E). ${ }^{38,40,42,43}$ To this end, KG-1a cells were labeled and subjected to analysis using the FMCR assay at varying shear stresses $\left(2,5,7,10,15\right.$ and 20 dynes $\left./ \mathrm{cm}^{2}\right)$ introduced at 30-second intervals (Figure S3A,B). Likewise, the total rolling duration at all tested shear force intervals showed similar uniformity across different UFIs $(70 \pm 57.82 \mathrm{~s})$ as shown by $\operatorname{ANOVA}(\mathrm{F}(6 ; 1017)=0.79 ; P=$ 0.578) and Tukey's posthoc test (Figure S3C; Table S1). The total number of rolling cells among the UFIs $(48.76 \pm 17.08$ cells $)$ were invariant as shown by ANOVA $(\mathrm{F}(6 ; 14)=0.792 ; P=0.591)$ and Tukey's post-hoc test (Figure S3D; Table S2). However, the mean rolling velocity showed some differences at 7 and 10 dyne/ $\mathrm{cm}^{2}$ (Figure S3E; Tables S9-S14). The $k_{\text {on }}$ of the interaction of selectin ligands with E-selectin is relatively slow ${ }^{40}$ and therefore the likelihood of making interactions is higher at lower shear stress compared to higher shear stresses. The slight variation observed at higher shear stresses may be due to the more erratic interactions of the cells with the E-selectin. Indeed, more variation is often observed with KGla cells using the conventional parallel plate flow chamber assay that is even more pronounced at higher shear stresses $33,38,43$ but may be underrepresented due to experimental bias. We analyzed the variation among the UFIs group means of the number of rolling cells per each shear stress step from the three replicated experiments using ANOVA followed by posthoc Tukey's multiple pairwise comparison test. ANOVA showed that there is no variation among the groups $(\mathrm{F}(30 ; 84)=0.249 ; P=0.999)$ and the post-hoc test (Figure S3F; Tables S3-S8).

\section{Case studies}

In one example, we assessed the dependence of E-selectin ligands on glycosylation for interacting 
with E-selectin by characterizing the rolling behavior of a number of deglycosylation treatments of KG-1a cells using the FMCR assay. KG-1a cells were treated with O-sialoglycoprotein endopeptidase (OSGE), peptide:N-glycosidase (PNGase-F) or $V$. cholerae neuraminidase (sialidase) enzymes to remove O-linked glycan chains, N-linked glycan chains or sialic acid respectively (see "Enzyme Treatment" in Methods of the Supporting Information file).$^{40}$ It is widely known that the disruption of surface glycans affects the E-selectin binding abilities as the E-selectin-binding motif, sialyl-Lewis $\mathrm{X}\left(\mathrm{sLe}^{\mathrm{x}}\right)$, can be expressed on O-linked or N-linked glycoproteins. ${ }^{38,40,41}$ Disruption of sialic acid affects E-selectin as well since sialic acid is an indispensable motif for sLe ${ }^{\mathrm{x}} / \mathrm{E}$-selectin interaction. ${ }^{40}$ Following the confirmation of enzymatic treatment (data not shown), each cell population was uniquely stained with an UFI. By running duplicate samples, we allowed for lower experimental variation through internal replication. Note that depending on the number of different samples run at a given time in a particular experiment (eg. one control and one treatment), additional replicates can be run (eg. triplicate of control with triplicate of treated) at the same time with differing fluorophores.

Untreated, OSGE-treated, PNGase-F-treated and sialidase-treated KG-1a cells were run under a series of 30-second shear stress steps ranging from 2 to $20 \mathrm{dyne} / \mathrm{cm}^{2}$ (Figure S4). Glycan disruption affected the mean rolling duration of all the rolling events significantly $(\mathrm{F}(3 ; 16)=8.965 ; P=0.001$; Figure 2A). The decrease in rolling duration was most apparent in the sialidase treated samples ( $\sim 10 \mathrm{X}$ less than untreated samples) while in the case of the OSGE and the PNGase-F the reduction was similar ( $\sim 2 \mathrm{X}$ less than the untreated samples). Analogously, the total number of rolling cells changed significantly upon deglycosylation $(\mathrm{F}(3 ; 16)=11.71 ; P=0.00026$; Figure 2B). The loss of rolling cells was highly significant in OSGE and PNGase-F ( 3.3X less) while more significant in sialidase treatment $(\sim 30 \mathrm{X}$ less). The mean velocity of rolling cells at different shear stress values were generally higher in the OSGE-treated and the PNGase-F-treated conditions due to the loss of the binding capacity following the removal of $\mathrm{O}$ - or $\mathrm{N}$-glycans (and hence $\mathrm{sLe}^{\mathrm{x}}$ epitopes on those 
branches) (Figure 2C). Although the $\mathrm{sLe}^{\mathrm{x}}$ structures were available on the remaining N-or O-glycans accordingly, there was a much more reduced number remaining and able to make the necessary binding interactions to the underlying E-selectin and thus resulted in higher velocity. ${ }^{40,42}$ As expected, due to the complete loss of sLe $e^{\mathrm{x}}$ epitopes necessary to make the adhesive interactions with E-selectin, the mean velocity of sialidase treated cells could not be determined. Finally, we found that the difference in the number of rolling cells over different magnitudes of shear stress was significantly different in all the deglycosylation treatments (Figure 2D) approaching very minimal numbers (close to zero) at higher shear stresses. This much faster decay in numbers of rolling cells following deglycosylation further recapitulates the dependency of $\mathrm{sLe}^{\mathrm{x}}$ structures to the maintenance of rolling and its resilience at higher shear stresses. ${ }^{40}$

To further illustrate the glycan-dependent nature of E-selectin binding to its ligands, in another example we created $\mathrm{sLe}^{\mathrm{x}}$ structures, using ex vivo fucosylation, on cells lacking them (data not shown) in order to improve their tethering and rolling interactions in a competitive setting on E-selectin expressing cells. These data are described in "Case Study II" in Supporting Information. 


\section{CONCLUSION}

In contrast to previous studies that showed inter-experimental variations using the standard PPFC assay, ${ }^{38-41}$ we believe the FMCR assay and analysis pipeline has eliminated this variation between samples and the need for normalization via running multiple samples side by side. The improved statistical power and the elimination of inter-experimental variation will help enhance the sensitivity of the assay to detect differences in rolling behavior, which could have significant consequences and lead to discoveries of biologically relevant phenomena. 


\section{ACKNOWLEDGMENTS}

We would like to thank Ms. Samar A. Rustum and Ms. Umme Habiba for their support in the management of the lab along with the entire Cell Migration and Signaling Laboratory for support and discussions. This research was supported by a King Abdullah University of Science and Technology (KAUST) Faculty Baseline Research Funding Program to J.S.M.

\section{AUTHOR CONTRIBUTION}

A.F.A. conceived, designed, performed and analyzed experiments and wrote the manuscript. A.A. designed, performed and analyzed experiments related to the recombinant human fucosyltransferase treatment. A.J.A. aided in conceiving the study. J.S.M. supervised and helped conceive the study as well as wrote the manuscript.

\section{CONFLICT OF INTEREST DISCLOSURE}

One or more US or international patent applications submitted by the authors relate to this work.

\section{SUPPORTING INFORMATION}

Methods and Analysis schemes, Supporting Figures (Figures S1-S6) and Supporting Tables (Tables S1-S14) are included in the "Supporting Information" file. 


\section{REFERENCES}

1. Horwitz, A. R., The origins of the molecular era of adhesion research. Nat Rev Mol Cell Biol 2012, 13 (12), 805-11.

2. Behrens, J., The role of cell adhesion molecules in cancer invasion and metastasis. Breast Cancer Res Treat 1993, 24 (3), 175-84.

3. Kolaczkowska, E.; Kubes, P., Neutrophil recruitment and function in health and inflammation. Nat Rev Immunol 2013, 13 (3), 159-75.

4. Leick, M.; Azcutia, V.; Newton, G.; Luscinskas, F. W., Leukocyte recruitment in inflammation: basic concepts and new mechanistic insights based on new models and microscopic imaging technologies. Cell Tissue Res 2014, 355 (3), 647-56.

5. Ley, K.; Laudanna, C.; Cybulsky, M. I.; Nourshargh, S., Getting to the site of inflammation: the leukocyte adhesion cascade updated. Nat Rev Immunol 2007, 7 (9), 678-89.

6. Reymond, N.; d'Agua, B. B.; Ridley, A. J., Crossing the endothelial barrier during metastasis. Nat Rev Cancer 2013, 13 (12), 858-70.

7. Lavdas, A. A.; Papastefanaki, F.; Thomaidou, D.; Matsas, R., Cell adhesion molecules in gene and cell therapy approaches for nervous system repair. Curr Gene Ther 2011, 11 (2), 90-100.

8. Merzaban, J. S.; Imitola, J.; Starossom, S. C.; Zhu, B.; Wang, Y.; Lee, J.; Ali, A. J.; Olah, M.; Abuelela, A. F.; Khoury, S. J.; Sackstein, R., Cell surface glycan engineering of neural stem cells augments neurotropism and improves recovery in a murine model of multiple sclerosis. Glycobiology 2015, 25 (12), 1392-409.

9. Sackstein, R.; Merzaban, J. S.; Cain, D. W.; Dagia, N. M.; Spencer, J. A.; Lin, C. P.; Wohlgemuth, R., Ex vivo glycan engineering of CD44 programs human multipotent mesenchymal stromal cell trafficking to bone. Nat Med 2008, 14 (2), 181-7.

10. Brown, D. C.; Larson, R. S., Improvements to parallel plate flow chambers to reduce reagent and cellular requirements. BMC Immunol 2001, $2,9$.

11. Lawrence, M. B.; McIntire, L. V.; Eskin, S. G., Effect of flow on polymorphonuclear leukocyte/endothelial cell adhesion. Blood 1987, 70 (5), 1284-90.

12. Sperandio, M.; Pickard, J.; Unnikrishnan, S.; Acton, S. T.; Ley, K., Analysis of leukocyte rolling in vivo and in vitro. Methods Enzymol 2006, 416, 346-71.

13. Stamper, H. B., Jr.; Woodruff, J. J., An in vitro model of lymphocyte homing. I. Characterization of the interaction between thoracic duct lymphocytes and specialized highendothelial venules of lymph nodes. J Immunol 1977, 119 (2), 772-80.

14. Gakhar, G.; Bander, N. H.; Nanus, D. M., In vitro method to observe E-selectin-mediated interactions between prostate circulating tumor cells derived from patients and human endothelial cells. J Vis Exp 2014, (87).

15. Gakhar, G.; Navarro, V. N.; Jurish, M.; Lee, G. Y.; Tagawa, S. T.; Akhtar, N. H.; Seandel, M.; Geng, Y.; Liu, H.; Bander, N. H.; Giannakakou, P.; Christos, P. J.; King, M. R.; Nanus, D. M., Circulating tumor cells from prostate cancer patients interact with E-selectin under physiologic blood flow. PLoS One 2013, 8 (12), e85143.

16. Carlson, G. E.; Martin, E. W.; Burdick, M. M., Simultaneously capturing real-time images in two emission channels using a dual camera emission splitting system: applications to cell adhesion. $J$ Vis Exp 2013, (79).

17. Merzaban, J. S.; Zuccolo, J.; Corbel, S. Y.; Williams, M. J.; Ziltener, H. J., An alternate core 2 beta1,6-N-acetylglucosaminyltransferase selectively contributes to P-selectin ligand formation in activated CD8 T cells. J Immunol 2005, 174 (7), 4051-9. 
18. Cumashi, A.; Ushakova, N. A.; Preobrazhenskaya, M. E.; D'Incecco, A.; Piccoli, A.; Totani, L.; Tinari, N.; Morozevich, G. E.; Berman, A. E.; Bilan, M. I.; Usov, A. I.; Ustyuzhanina, N. E.; Grachev, A. A.; Sanderson, C. J.; Kelly, M.; Rabinovich, G. A.; lacobelli, S.; Nifantiev, N. E.; Consorzio Interuniversitario Nazionale per la Bio-Oncologia, I., A comparative study of the anti-inflammatory, anticoagulant, antiangiogenic, and antiadhesive activities of nine different fucoidans from brown seaweeds. Glycobiology 2007, 17 (5), 541-52.

19. Descheny, L.; Gainers, M. E.; Walcheck, B.; Dimitroff, C. J., Ameliorating skin-homing receptors on malignant $\mathrm{T}$ cells with a fluorosugar analog of $\mathrm{N}$-acetylglucosamine: $\mathrm{P}$-selectin ligand is a more sensitive target than E-selectin ligand. J Invest Dermatol 2006, 126 (9), 2065-73.

20. Cedeno-Laurent, F.; Opperman, M. J.; Barthel, S. R.; Hays, D.; Schatton, T.; Zhan, Q.; He, X.; Matta, K. L.; Supko, J. G.; Frank, M. H.; Murphy, G. F.; Dimitroff, C. J., Metabolic inhibition of galectin-1-binding carbohydrates accentuates antitumor immunity. J Invest Dermatol 2012, 132 (2), 410-20.

21. Levy, O.; Mortensen, L. J.; Boquet, G.; Tong, Z.; Perrault, C.; Benhamou, B.; Zhang, J.; Stratton, T.; Han, E.; Safaee, H.; Musabeyezu, J.; Yang, Z.; Multon, M. C.; Rothblatt, J.; Deleuze, J. F.; Lin, C. P.; Karp, J. M., A small-molecule screen for enhanced homing of systemically infused cells. Cell Rep 2015, 10 (8), 1261-1268.

22. Xu, Y.; Wang, B.; Deng, J.; Liu, Z.; Zhu, L., A potential model for drug screening by simulating the effect of shear stress in vivo on endothelium. Biorheology 2013, 50 (1-2), 33-43.

23. Jiang, X. Y.; Sarsons, C. D.; Gomez-Garcia, M. J.; Cramb, D. T.; Rinker, K. D.; Childs, S. J., Quantum dot interactions and flow effects in angiogenic zebrafish (Danio rerio) vessels and human endothelial cells. Nanomedicine 2017, 13 (3), 999-1010.

24. Dong, C.; Lei, X. X., Biomechanics of cell rolling: shear flow, cell-surface adhesion, and cell deformability. J Biomech 2000, 33 (1), 35-43.

25. Lee, C. H.; Bose, S.; Van Vliet, K. J.; Karp, J. M.; Karnik, R., Studying cell rolling trajectories on asymmetric receptor patterns. $J$ Vis Exp 2011, (48).

26. Lee, C. H.; Bose, S.; Van Vliet, K. J.; Karp, J. M.; Karnik, R., Examining the lateral displacement of HL60 cells rolling on asymmetric P-selectin patterns. Langmuir 2011, 27 (1), 240-9.

27. Levy, O.; Anandakumaran, P.; Ngai, J.; Karnik, R.; Karp, J. M., Systematic analysis of in vitro cell rolling using a multi-well plate microfluidic system. $J$ Vis Exp 2013, (80), e50866.

28. Ling, X.; Ye, J. F.; Zheng, X. X., Dynamic investigation of leukocyte-endothelial cell adhesion interaction under fluid shear stress in vitro. Sheng Wu Hua Xue Yu Sheng Wu Wu Li Xue Bao (Shanghai) 2003, 35 (6), 567-72.

29. Sackstein, R.; Fuhlbrigge, R., The blot rolling assay: a method for identifying adhesion molecules mediating binding under shear conditions. Methods Mol Biol 2006, 341, 217-26.

30. Shetty, S.; Weston, C. J.; Adams, D. H.; Lalor, P. F., A flow adhesion assay to study leucocyte recruitment to human hepatic sinusoidal endothelium under conditions of shear stress. $J$ Vis Exp 2014, (85).

31. Shetty, S.; Bruns, T.; Weston, C. J.; Stamataki, Z.; Oo, Y. H.; Long, H. M.; Reynolds, G. M.; Pratt, G.; Moss, P.; Jalkanen, S.; Hubscher, S. G.; Lalor, P. F.; Adams, D. H., Recruitment mechanisms of primary and malignant B cells to the human liver. Hepatology 2012, 56 (4), 1521-31.

32. Usami, S.; Chen, H. H.; Zhao, Y.; Chien, S.; Skalak, R., Design and construction of a linear shear stress flow chamber. Ann Biomed Eng 1993, 21 (1), 77-83.

33. Wiese, G.; Barthel, S. R.; Dimitroff, C. J., Analysis of physiologic E-selectin-mediated leukocyte rolling on microvascular endothelium. J Vis Exp 2009, (24). 
34. Tsurui, H.; Nishimura, H.; Hattori, S.; Hirose, S.; Okumura, K.; Shirai, T., Seven-color fluorescence imaging of tissue samples based on Fourier spectroscopy and singular value decomposition. J Histochem Cytochem 2000, 48 (5), 653-62.

35. Krutzik, P. O.; Nolan, G. P., Fluorescent cell barcoding in flow cytometry allows highthroughput drug screening and signaling profiling. Nat Methods 2006, 3 (5), 361-8.

36. Krutzik, P. O.; Clutter, M. R.; Trejo, A.; Nolan, G. P., Fluorescent cell barcoding for multiplex flow cytometry. Curr Protoc Cytom 2011, Chapter 6, Unit 631.

37. Krutzik, P. O.; Nolan, G. P., Intracellular phospho-protein staining techniques for flow cytometry: monitoring single cell signaling events. Cytometry A 2003, 55 (2), 61-70.

38. AbuSamra, D. B.; Aleisa, F. A.; Al-Amoodi, A. S.; Jalal Ahmed, H. M.; Chin, C. J.; Abuelela, A. F.; Bergam, P.; Sougrat, R.; Merzaban, J. S., Not just a marker: CD34 on human hematopoietic stem/progenitor cells dominates vascular selectin binding along with CD44. Blood Adv 2017, 1 (27), 2799-2816.

39. Aleisa, F. A.; Sakashita, K.; Lee, J. M.; AbuSamra, D. B.; Al Alwan, B.; Nozue, S.; Tehseen, M.; Hamdan, S. M.; Habuchi, S.; Kusakabe, T.; Merzaban, J. S., Functional binding of E-selectin to its ligands is enhanced by structural features beyond its lectin domain. The Journal of biological chemistry 2020, 295 (11), 3719-3733.

40. AbuSamra, D. B.; Al-Kilani, A.; Hamdan, S. M.; Sakashita, K.; Gadhoum, S. Z.; Merzaban, J. S., Quantitative Characterization of E-selectin Interaction with Native CD44 and P-selectin Glycoprotein Ligand-1 (PSGL-1) Using a Real Time Immunoprecipitation-based Binding Assay. J Biol Chem 2015, 290 (35), 21213-30.

41. Ali, A. J.; Abuelela, A. F.; Merzaban, J. S., An Analysis of Trafficking Receptors Shows that CD44 and P-Selectin Glycoprotein Ligand-1 Collectively Control the Migration of Activated Human TCells. Front Immunol 2017, 8, 492.

42. AbuZineh, K.; Joudeh, L. I.; Al Alwan, B.; Hamdan, S. M.; Merzaban, J. S.; Habuchi, S., Microfluidics-based super-resolution microscopy enables nanoscopic characterization of blood stem cell rolling. Sci Adv 2018, 4 (7).

43. Merzaban, J. S.; Burdick, M. M.; Gadhoum, S. Z.; Dagia, N. M.; Chu, J. T.; Fuhlbrigge, R. C.; Sackstein, R., Analysis of glycoprotein E-selectin ligands on human and mouse marrow cells enriched for hematopoietic stem/progenitor cells. Blood 2011, 118 (7), 1774-1783. 


\section{FIGURES}

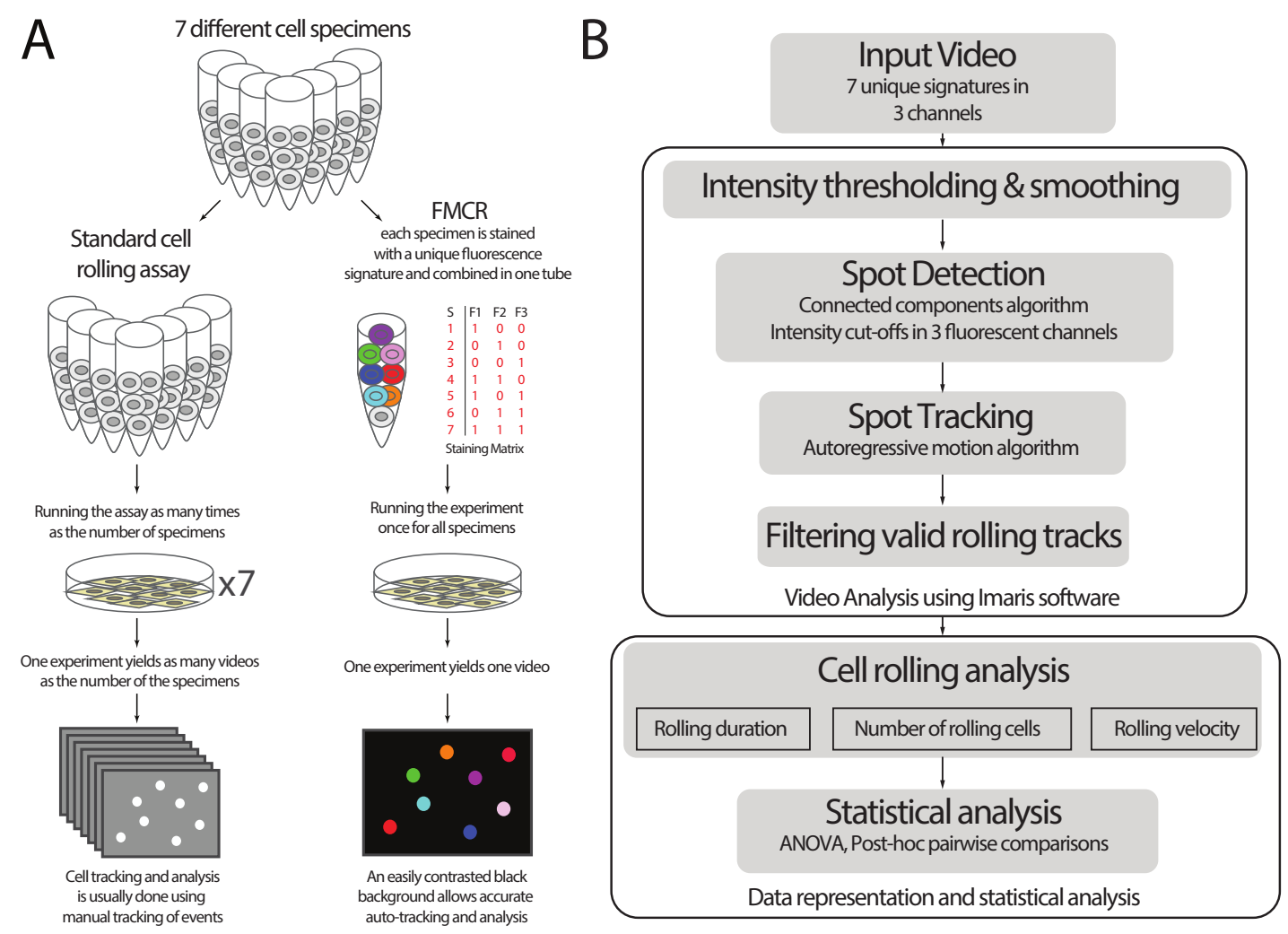

Figure 1. Experimental outline for the FMCR. (A) Comparison of the work-flow of the conventional parallel plate flow assay (left) and the FMCR assay (right). For one biological replicate $n=1$ of a hypothetical flow assay of $s=7$ different samples, the conventional approach implies running a number of experiments equal to the number of samples (s). Also, it implies the use of s plates of the substrate-expressing cells and producing s videos for subsequent analysis. The FMCR approach uses a combination of three fluorophores to produce 7 UFIs using the staining matrix to tag the samples. This implies the use of one plate of the substrate-expressing cells and the production of one video for subsequent analysis. (B) The analysis pipeline developed for the analysis of the time-lapse FMCR assay runs. The pipeline uses the raw videos as input. It has two phases: (1) using the Imaris software for cell detection and tracking, and (2) data representation and statistical analysis using the R statistical tool bundle. 

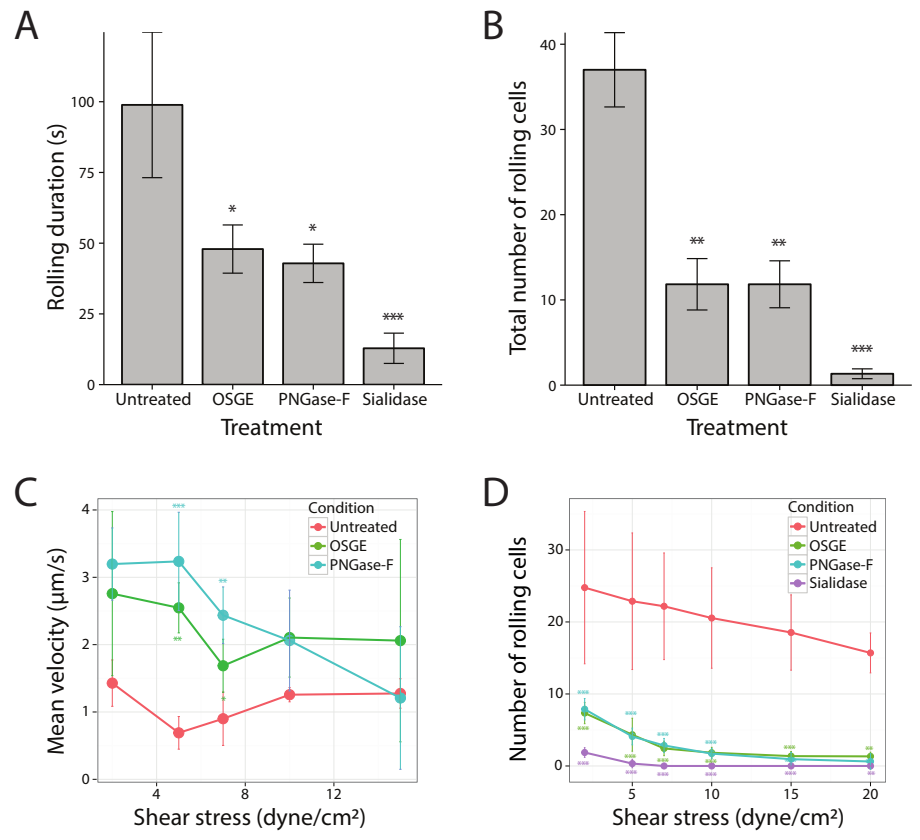

Figure 2. Case study highlighting how manipulation of glycosylation of hematopoietic cells affects their rolling over E-selectin-expressing CHO cells. (A-D) Sample experiment highlighting how deglycosylation (OSGE, PNGase-F, sialidase) treatment of KG-1a cells disturbs rolling over Eselectin-expressing $\mathrm{CHO}$ cells. (A) The average rolling duration from 3 independent experiments at increasing shear stress intervals. (B) The total number of rolling cells. (C) The mean velocity per shear stress step from three independent experiments. (D) The mean number of rolling cells per shear stress step from three independent experiments. Data are represented as the mean \pm SD $(\mathrm{n}=3$ independent experiments; $(*) P \leq 0.01,(* *) P \leq 0.001,(* * *) P \leq 0.0001$.

\section{FOR TABLE OF CONTENTS ONLY GRAPHIC}

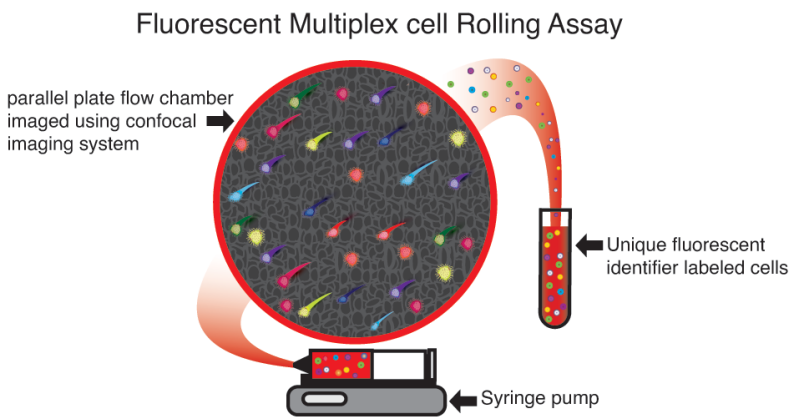




\title{
Supporting Information
}

\section{Fluorescent multiplex cell rolling assay: simultaneous capturing up to seven samples in real-time using spectral confocal microscopy}

\author{
Ayman F. AbuElela, Asma S. Al-Amoodi, Amal J. Ali and Jasmeen S. Merzaban* \\ Cell Migration and Signaling Laboratory, King Abdullah University of Science and Technology, \\ Biological and Environmental Sciences and Engineering Division, Thuwal, Saudi Arabia
}

* Correspondence:

Jasmeen S. Merzaban

4700 King Abdullah University of Science and Technology

Thuwal, Saudi Arabia

$23955-6900$

jasmeen.merzaban@kaust.edu.sa 


\section{TABLE OF CONTENTS}

CASE STUDY II

METHODS

Preparation of substrate-expressing cells for FMCR:

Fluorescent activated cell sorting (FACS):

Data representation and statistical analysis using the $\mathrm{R}$ software package:

Enzyme Treatment:

Hypothesis Testing:

FIGURES

Figure S1. Schematic illustration of the PPFC setup for the FMCR.

Figure S2. Labeling of KG1a cells using a combination of three fluorophores to form seven distinctive UFIs.

Figure S3. Detection of fluorescence tagging bias using seven identical samples of KG-1a cells.

Figure S4. Sample experiment highlighting how deglycosylation (OSGE, PNGase-F, sialidase) treatment of KG-1a cells disturbs rolling over E-selectin-expressing CHO cells.

Figure S5. Sample experiment highlighting how the addition of fucose by rhFTVI to create $\mathrm{sLe}^{\mathrm{x}}$ structures on $\mathrm{K} 562$ cells is able to enhance their ability to roll over E-selectin-expressing $\mathrm{CHO}$ cells in a competitive manner.

Figure S6. Case study highlighting how manipulation of glycosylation using ex vivo fucosyltransferase treatment of hematopoietic cells enhances their rolling over E-selectin-expressing $\mathrm{CHO}$ cells.

TABLES.

Table S1: Tukey's honest significant difference (HSD) multiple pairwise comparison analysis of the rolling duration between different UFI-tagged KG-1a cells. 
Table S2: Tukey's HSD multiple pairwise comparison analysis of the mean number of rolling cells between different UFI-tagged KG-1a cells.

Table S3: Tukey's HSD multiple pairwise comparison analysis of the mean number of rolling cells between different UFI-tagged KG-1a cells at 2 dyne $/ \mathrm{cm}^{2}-\mathrm{F}(6 ; 14=0: 673 ; \mathrm{p}=0: 674)$.

Table S4: Tukey's HSD multiple pairwise comparison analysis of the mean number of rolling cells between different UFI-tagged KG-1a cells at 5 dyne $/ \mathrm{cm}^{2}-\mathrm{F}(6 ; 14=1: 205 ; \mathrm{p}=0: 36)$.

Table S5: Tukey's HSD multiple pairwise comparison analysis of the mean number of rolling cells between different UFI-tagged KG-1a cells at 7 dyne $/ \mathrm{cm}^{2}-\mathrm{F}(6 ; 14=0: 851 ; \mathrm{p}=0: 553)$.

Table S6: Tukey's HSD multiple pairwise comparison analysis of the mean number of rolling cells between different UFI-tagged KG-1a cells at 10 dyne $/ \mathrm{cm}^{2}-\mathrm{F}(6 ; 14=0: 358 ; \mathrm{p}=0: 893)$

Table S7: Tukey's HSD multiple pairwise comparison analysis of the mean number of rolling cells between different UFI-tagged KG-1a cells at 15 dyne $/ \mathrm{cm}^{2}-\mathrm{F}(6 ; 14=0: 454 ; \mathrm{p}=0: 831)$

Table S8: Tukey's HSD multiple pairwise comparison analysis of the mean number of rolling cells between different UFI-tagged KG-1a cells at 20 dyne $/ \mathrm{cm}^{2}-\mathrm{F}(6 ; 14=1: 258 ; \mathrm{p}=0: 337)$

Table S9: Tukey's HSD multiple pairwise comparison analysis of the velocity of rolling cells between different UFI-tagged KG-1a cells at 2 dyne $/ \mathrm{cm}^{2}-\mathrm{F}(6 ; 14=0: 575 ; \mathrm{p}=0: 744)$.

Table S10: Tukey's HSD multiple pairwise comparison analysis of the velocity of rolling cells between different UFI-tagged KG-1a cells at 5 dyne $/ \mathrm{cm}^{2}-\mathrm{F}(6 ; 14=0: 796 ; \mathrm{p}=0: 588)$

Table S11: Tukey's HSD multiple pairwise comparison analysis of the velocity of rolling cells between different UFI-tagged KG-1a cells at 7 dyne $/ \mathrm{cm}^{2}-\mathrm{F}(6 ; 14=3: 296 ; \mathrm{p}=0: 0309)$..... S-26

Table S12: Tukey's HSD multiple pairwise comparison analysis of the velocity of rolling cells between different UFI-tagged KG-1a cells at 10 dyne $/ \mathrm{cm}^{2}-\mathrm{F}(6 ; 14=3: 421 ; \mathrm{p}=0: 0271)$

Table S13: Tukey's HSD multiple pairwise comparison analysis of the velocity of rolling cells between different UFI-tagged KG-1a cells at 15 dyne $/ \mathrm{cm}^{2}-\mathrm{F}(6 ; 14=1: 231 ; \mathrm{p}=0: 348)$ 
Table S14: Tukey's HSD multiple pairwise comparison analysis of the velocity of rolling cells between different UFI-tagged KG-1a cells at 20 dyne $/ \mathrm{cm}^{2}-\mathrm{F}(6 ; 14=1: 399 ; \mathrm{p}=0: 282)$....................................

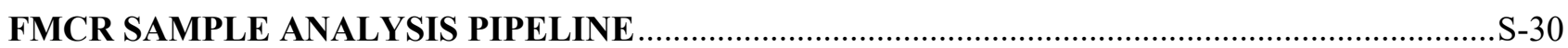

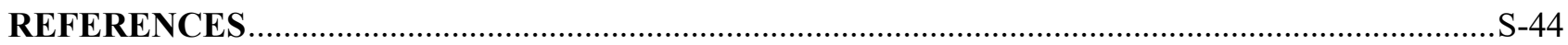




\section{CASE STUDY II}

Selectins bind to sialofucosylated glycans, specifically the tetrasaccharide sialyl Lewis $\mathrm{X}\left(\mathrm{sLe}^{\mathrm{x}}\right.$ ), on glycoproteins or glycolipids. sLe ${ }^{\mathrm{x}}$-modified glycoproteins such as CD44, PSGL-1, CD43 and CD34 bind Eselectin, ${ }^{1-3}$ but expression of these glycoproteins alone does not predict whether E-selectin will bind. K562 cells, previously determined to neither express $\mathrm{SLe}^{\mathrm{x}}$ epitopes nor to bind E-selectin (data not shown), gained $\mathrm{sLe}^{\mathrm{x}}$ expression and E-selectin binding following treatment with recombinant human fucosyltransferase VI (rhFTVI), an enzyme that adds the essential fucose residues to create $\mathrm{sLe}^{\mathrm{x}}$ structures from sialylated precursors (data not shown) as previously reported for mesenchymal stromal cells and neural stem cells. ${ }^{4,5}$

Using FTVI-treated and untreated K562 cells (see "Enzyme Treatment" in Methods below), we compared their rolling behavior using our FMCR assay. Previous studies showed that cells with poor binding activity to Eselectin could be enhanced through fucosylation and made to roll on independent E-selectin expressing surfaces. ${ }^{4,5}$ Here, instead of performing the rolling studies separately, i.e. in independent runs, we directly measured th e ability of treated and untreated K562 cells along with KG1a cells (positive control) to compete on the same E-selectin expressing surface (i.e. CHO-E cells) using the FMCR assay. To this end, following the treatment of K562 cells with rhFTVI, each cell population was labeled with two different UFIs (Figure S5). We allowed the mixture of cells (uniquely labeled KG1a cells, buffer-treated K562 cells and rhFTVI-treated K562 cells) to bind to E-selectin expressing cells (CHO-E) for 2 minutes at 0.2 dyne $/ \mathrm{cm}^{2}$ (Figure S5). In a stepwise manner, as described in the main text, we increased the shear stress from $1-10 \mathrm{dyne} / \mathrm{cm}^{2}$ at 30 second intervals programmed by the syringe pump. As illustrated in Figure S6A and S6B, rhFTVI-treated cells interacted and rolled on the CHO-E monolayer with higher frequency than buffered-treated samples did as represented by a 2 -fold higher average rolling duration $(n=3$ independent experiments; $P=0.0093)$ and a 3.5 fold higher total number of rolling cells over all shear stresses $(n=3$ independent experiments; $P=0.0001)$ following treatment of the K562 cells with rhFTVI. Moreover, as presented in Figure S6C, the rolling velocity over the different shear stresses tested was higher in buffered-treated samples compared to the rhFTVI-treated samples and KG1a cells, which was more obvious in the lower shear stresses tested (1 and 2 dyne $\left./ \mathrm{cm}^{2}\right)$. Analysis of the number of rolling cells at the different shear stresses tested indicated that rhFTVI treatment of 
K562 cells resulted in similar numbers of rolling cells as KG1a cells (positive control) compared to buffertreated K562 cells (Figure S6D). These results indicate that fucosylation of cells improves their ability to compete with KG1a cells for E-selectin expressed on CHO-E cells.

This case study and the one presented in the main text where glycan structures on cell surface glycoproteins essential for binding to selectins are manipulated either by their specific removal or their addition by various enzymes not only recapitulated the importance of $\mathrm{sLe}^{\mathrm{x}}$ to the binding of cells to E-selectin, but also emphasized the role of sialic acid and fucose to the rolling interactions of cells on E-selectin expressing surfaces in a competitive manner for the first time. 


\section{METHODS}

\section{Preparation of substrate-expressing cells for FMCR:}

The PPFC assay requires preparing a plate seeded with a confluent monolayer of substrate expressing cells (e.g. CHO-E). To achieve this, the substrate-expressing cells were assessed for the expression of the substrate (e.g. E-selectin) before seeding using surface marker expression analysis via flow cytometry (or an equivalent technique; data not shown). Subsequently, the cells were then seeded on tissue culture-treated cover slips for an amount of time sufficient to form a confluent monolayer of cells and before reaching over-confluence (i.e. preferably between $70-90 \%$ confluency). We found that confluency was a critical factor as the inter-cellular spaces can heavily affect the rolling dynamics of the cells under flow, likely due to intermittent exposure to cell capturing substrate molecules. An assessment of cell growth kinetics was performed in order to determine the time needed to form the cell monolayer on cover slips (data not shown).

\section{Fluorescent activated cell sorting (FACS):}

Cells were harvested and $1-2 \times 10^{5}$ cells from each cell line or treatment condition were washed twice using 1X PBS before staining with the primary antibody of interest at $1: 100$ dilution and incubated at $4^{\circ} \mathrm{C}$ for 30 minutes in $1 \%$ FBS in HBSS (Gibco, Grand Island, NY, USA). Cells were washed twice and stained with the appropriate secondary antibody for 15 minutes at 1:200 dilution at $4^{\circ} \mathrm{C}$. Typically, anti-mouse IgG secondary antibodies conjugated with various fluorophores (AF405, AF546 and/or AF680) were used either alone or in combination (mixture of 2 or 3 fluorophores) to stain the primary antibodies at a concentration of $5 \mu \mathrm{g} / \mathrm{mL}$ for 15 minutes at $4^{\circ} \mathrm{C}$. Cells were analyzed using BD FACS CANTO II (BD Biosciences, San Jose, CA, USA). AF405 was excited by the $405 \mathrm{~nm}$ laser and detected by the $450 / 50$ filter, AF546 was excited by 488 $\mathrm{nm}$ laser and detected by 585/42 filter and AF680 was excited by a $633 \mathrm{~nm}$ laser and detected by a 780/60 filter. FACS data were analyzed using FlowJo (FlowJo LLC., Ashland, OR, USA) using the isotype antibody as a control. 


\section{Data representation and statistical analysis using the $R$ software package:}

We developed a comprehensive data analysis pipeline specifically designed for the output of the video analysis performed by the Imaris software (see "FMCR Sample Analysis Pipeline" below). It aimed to make use of the high temporal resolution and the high number of events needed to achieve high statistical power and sensitivity. The analysis focused on the rolling parameters affected by the adhesion kinetics such as the number of rolling cells, the rolling duration, the rolling displacement and the rolling velocity. The pipeline incorporates statistical analysis of multiple hypothesis testing and the post-hoc multiple comparisons.

\section{Enzyme Treatment:}

N-Linked glycoprotein was removed in KGla cells by treating the lysate with commercially available PNGase F (8 units/mL) diluted with G7 buffer (50 mM sodium phosphate (pH 7.5)) and 1\% Nonidet P-40 for $24 \mathrm{~h}$ at $37^{\circ} \mathrm{C}$. O-Glycans were removed by O-sialoglycoprotein endopeptidase (OSGE) $(240 \mu \mathrm{g} / \mathrm{mL})$ treatment for 4 $\mathrm{h}$ at $37^{\circ} \mathrm{C}$. OSGE was lyophilized in HEPES buffer (pH 7.4) and replenished in the same buffer containing $1 \%$ BSA. Sialic acid was removed by incubating $V$. cholerae neuraminidase $(0.2 \mathrm{unit} / \mathrm{mL})$ in $50 \mathrm{mM}$ sodium acetate buffer ( $\mathrm{pH}$ 5.5) containing $5 \mathrm{mM} \mathrm{CaCl}_{2}$ and $150 \mathrm{mM} \mathrm{NaCl}$ with lysate for $3 \mathrm{~h}$ at $37^{\circ} \mathrm{C}$. As a control for the treatment conditions, cells for each enzyme digestion were treated with the same buffer and incubated for the same duration and temperature but without the enzymes. For fucosyltransferase treatment, K562 cells were harvested, washed twice with Hank's Balanced Salt Solution (HBSS) and then resuspended at a density of $1 \times 10^{6}$ cells $/ \mathrm{mL}$ in recombinant human fucosyltransferase (rhFTVI; produced in our laboratory) reaction buffer [25 mM HEPES (pH 7.5) (Gibco Invitrogen), 0.1\% human serum albumin (Sigma-Aldrich), 0.5 mM GDPfucose (Sigma), $5 \mathrm{mM} \mathrm{MnCl}_{2}$ ] and appropriate amount of purified rhFTVI enzyme in HBSS and incubated at $37^{\circ} \mathrm{C}$ for 30 minutes. Buffer only controls without the rhFTVI enzyme were used as a negative control. The cells were then washed twice with HBSS and 10 mM EDTA.

\section{Hypothesis testing:}

Multiple hypothesis testing was performed using one-way analysis of variance (ANOVA) followed by multiple pairwise comparisons Tukey's honest significant difference (HSD) testing. Two-sample hypothesis testing was performed using Student's T-test. 


\section{FIGURES}

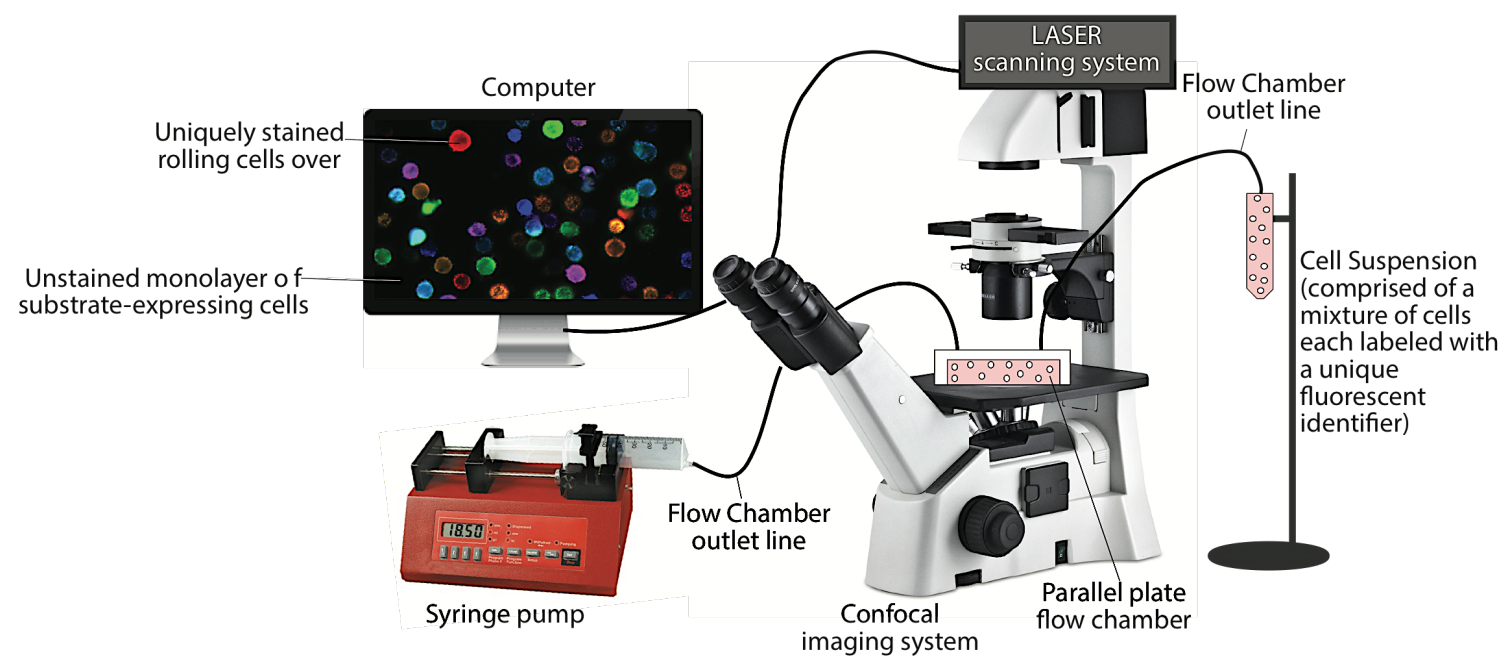

Figure S1. Schematic illustration of the PPFC setup for the FMCR.

A monolayer of ligand-expressing adherent cells is grown on a tissue culture-coated coverslip (eg. CHO-E cells). A PPFC is assembled and positioned on the stage of the confocal laser scanning system where the cell monolayer is kept in native culture conditions of medium and temperature. A mixture of labeled cell suspension is run through connecting tubes under controlled flow brought about by a programmable syringe pump. Rolling events are detected using a confocal laser scanning system in a time-series experiment. The system excites a combination of spectrally separated fluorophores simultaneously using single-wavelength laser lines. Emission signals denoting distinct cells are captured using a charge-coupled device (CCD) imaging camera. The timeseries experiment is exported for downstream analysis using flow-tracking software. 


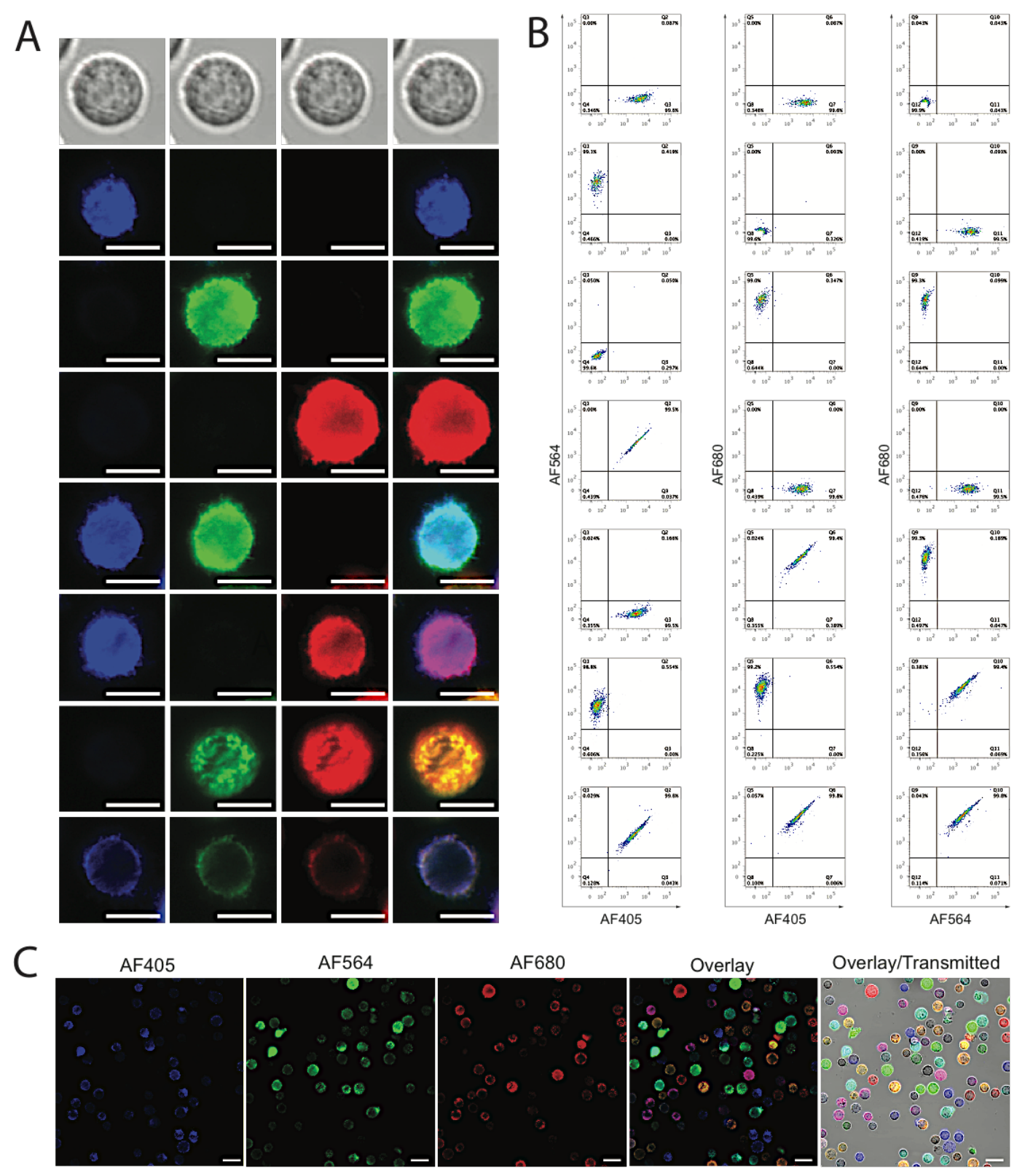

Figure S2. Labeling of KG1a cells using a combination of three fluorophores to form seven distinctive UFIs.

(A) Fluorescence images of the UFIs used to tag up to seven samples using a combination of three fluorophores: AF405, AF546 and AF680. (B) Confirmation of absence of the spectral overlap in combinatorial staining using uncompensated FACS analysis. (C) Example frame of the FMCR assay showing the mixture of seven samples tagged with 7 different UFIs. AF, Alexa-Fluor. Size bar $=10 \mu \mathrm{m}$. 


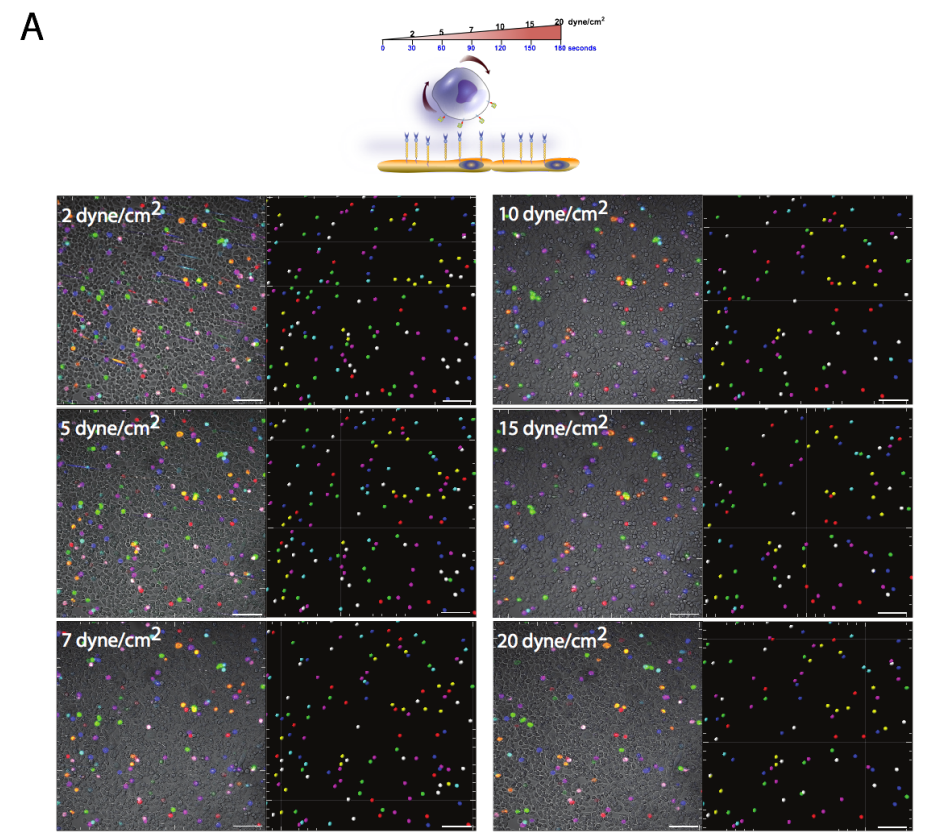

B
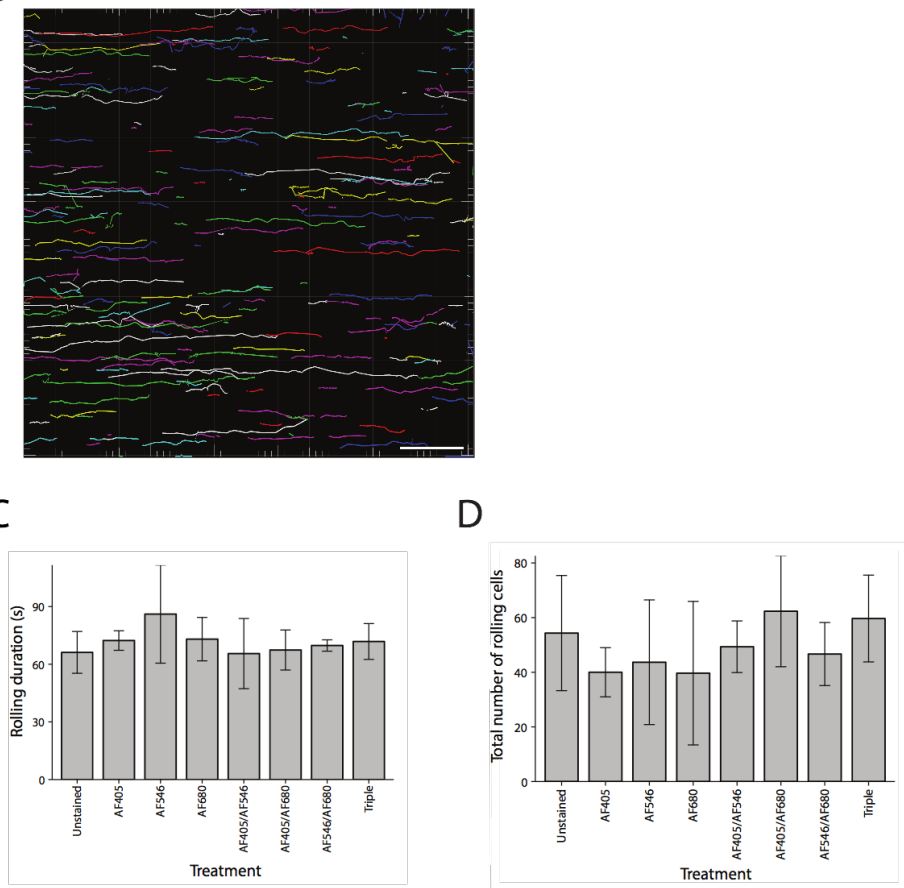

E

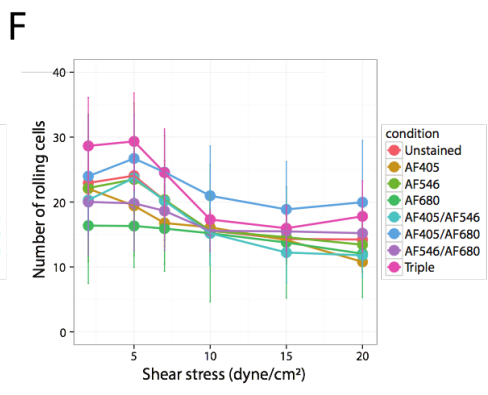


Figure S3. Detection of fluorescence tagging bias using seven identical samples of KG-1a cells.

(A) Model of experimental setup illustrating that every 30 -seconds the shear stress was varied starting from 2 dyne $/ \mathrm{cm}^{2}$ up to a maximum of $20 \mathrm{dyne} / \mathrm{cm}^{2}$ following a 2-minute flow at $0.2 \mathrm{dyne} / \mathrm{cm}^{2}$ in order to establish initial interactions of the cells with the E-selectin expressing monolayer. Representative screen-shots of the cells under flow shear stress magnitudes from 2 dyne $/ \mathrm{cm}^{2}$ to 20 dyne $/ \mathrm{cm}^{2}$ are shown. At each of the 30 -second shear stress step, a series of snapshots were taken; the raw image (left) is shown along with an image where the rolling events were reconstructed using the Imaris cell tracking software (right). (B) The software reconstruction of the rolling tracks. The track colors represent the rolling of the cells at increasing shear stress intervals of seven UFIs. (C) The rolling duration of the KG-1a cells tagged with seven different UFIs from 3 replicated experiments at increasing shear stress intervals. (D) The total number of rolling cells. (E) The mean velocity per shear stress step from three replicated experiments. (F) The mean number of rolling cells per shear stress step from three replicated experiments. Error bars represent the standard deviation (SD). Size bar $=10$ $\mu \mathrm{m}$. 
A
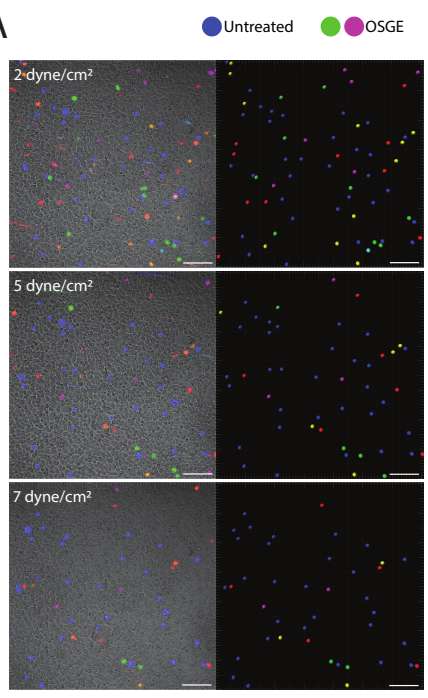

- PNGase-F OSialidase
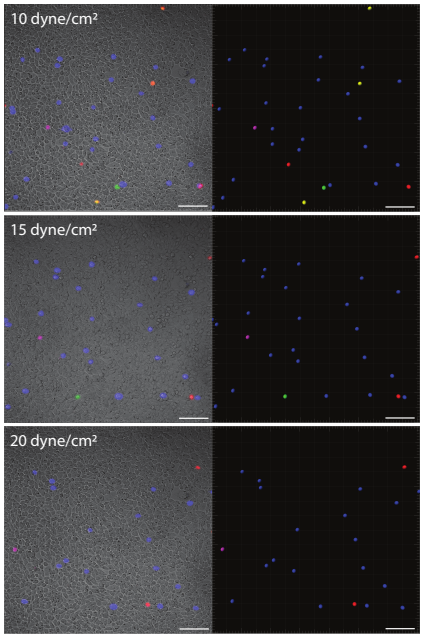

B

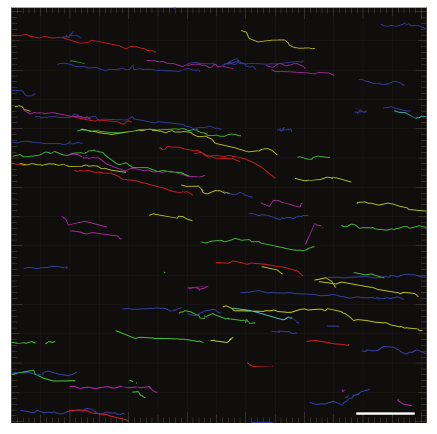

Figure S4. Sample experiment highlighting how deglycosylation (OSGE, PNGase-F, sialidase) treatment of KG-1a cells disturbs rolling over E-selectin-expressing CHO cells.

(A) Representative screen-shots of the cells under flow at each shear stress step from 2-20 dyne $/ \mathrm{cm}^{2}$ is shown. At each of the 30-second shear stress steps (Figure S3A, top), a series of snapshots were taken; the raw image is shown (left) along with an image where the rolling events were reconstructed using the Imaris cell tracking software (right). (B) The software reconstruction of the rolling tracks. The track colors represent the rolling of the cells at increasing shear stress intervals of seven UFIs. Size bar $=10 \mu \mathrm{m}$. 
A
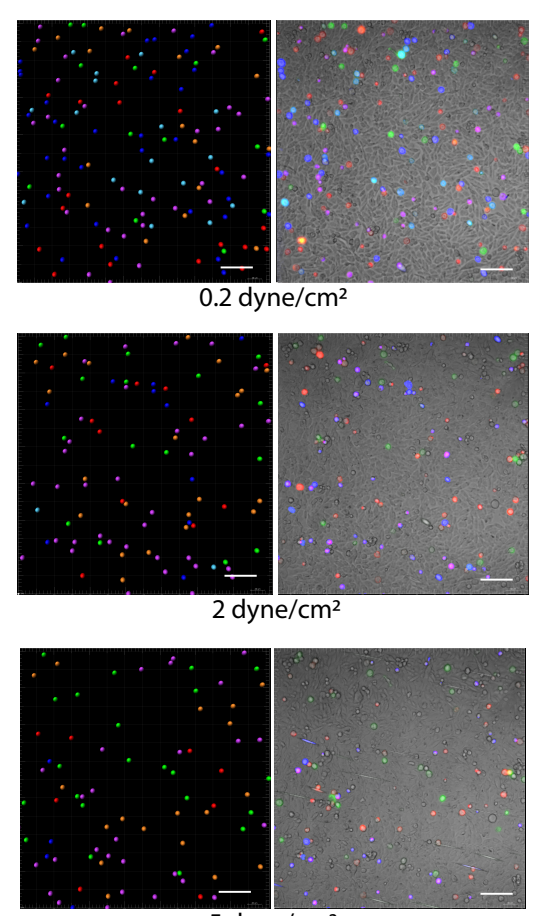

5 dyne $/ \mathrm{cm}^{2}$
B

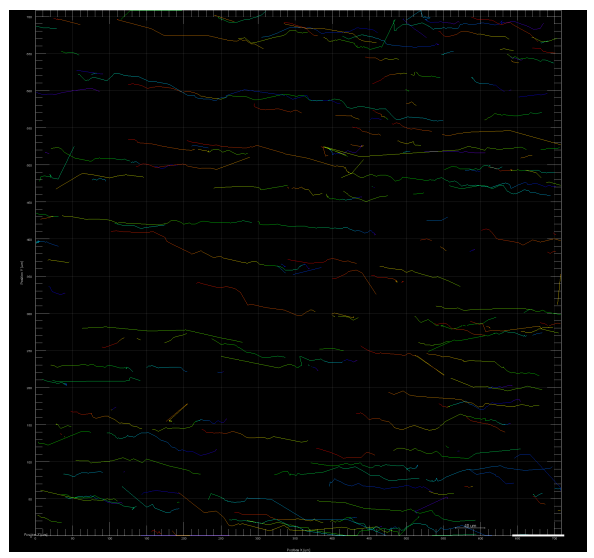

Figure S5. Sample experiment highlighting how the addition of fucose by rhFTVI to create sLe ${ }^{\mathrm{x}}$ structures on K562 cells is able to enhance their ability to roll over E-selectin-expressing CHO cells in a competitive manner.

(A) Representative screen-shots of cells under flow at 0.2 dyne $/ \mathrm{cm}^{2}, 2$ dyne $/ \mathrm{cm}^{2}$, and $5 \mathrm{dyne} / \mathrm{cm}^{2}$ is shown. At each of the 30-second shear stress steps (Figure S3A, top), a series of snapshots were taken; the raw image is shown (left) along with an image where the rolling events were reconstructed using the Imaris cell tracking software (right). Rolling interactions, captured using a confocal laser scanning system at a rate of 1 frame/second, at $0.2 \mathrm{dyne} / \mathrm{cm}^{2}, 2 \mathrm{dyne} / \mathrm{cm}^{2}$, and $5 \mathrm{dyne} / \mathrm{cm}^{2}$ are shown. (B) Cell tracking analysis was performed using the Imaris 8 software, which allowed for the reconstruction of the rolling tracks. The track colors represent the rolling of the cells at increasing shear stress intervals of six UFIs. Size bar $=10 \mu \mathrm{m}$. 
A

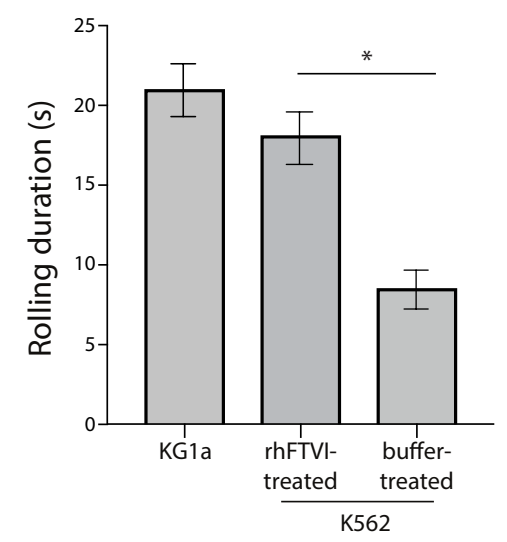

C

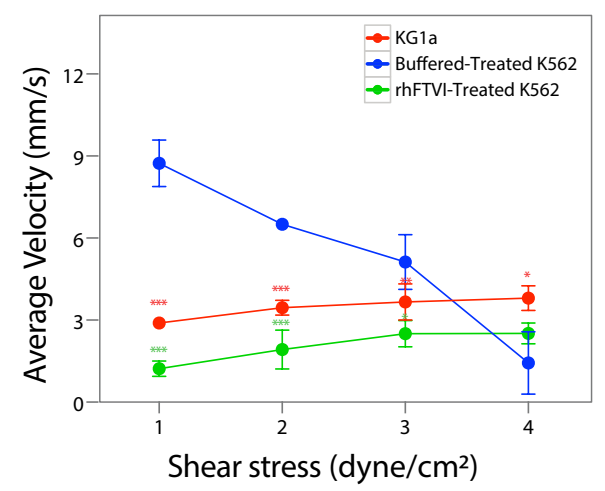

B

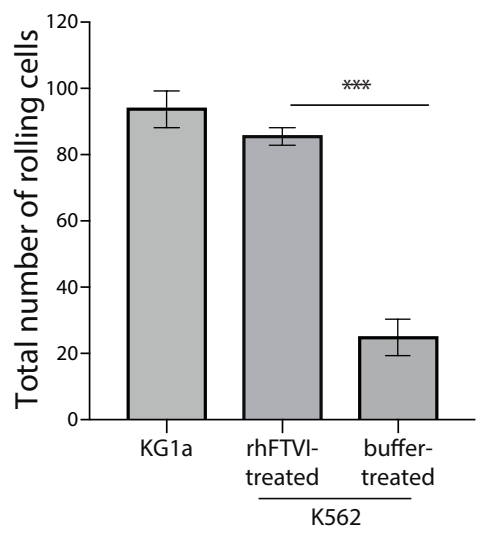

D

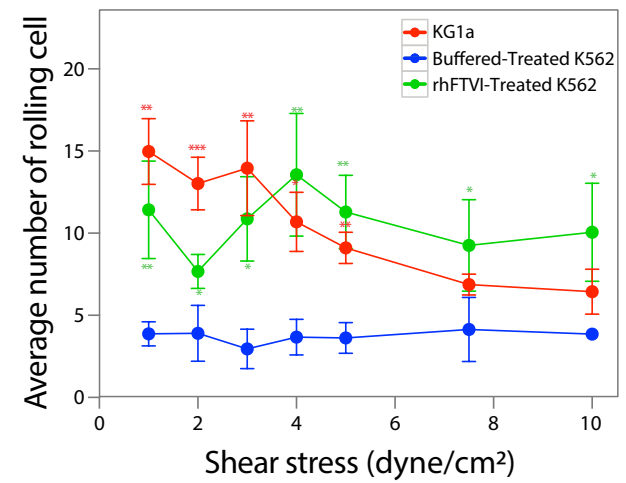

Figure S6. Case study highlighting how manipulation of glycosylation using ex vivo fucosyltransferase treatment of hematopoietic cells enhances their rolling over E-selectin-expressing $\mathrm{CHO}$ cells.

(A-D) Sample experiment highlighting how the addition of fucose by rhFTVI to create sLe ${ }^{\mathrm{x}}$ structures on K562 cells is able to enhance their ability to roll over E-selectin-expressing CHO cells in a competitive manner. (A) The average rolling duration from 3 independent experiments at increasing shear stress intervals. (B) The total number of rolling cells. (C) The velocity per shear stress step for three independent experiments. (D) The mean number of rolling cells per shear stress step from three independent experiments. Data are represented as the mean $\pm \mathrm{SD}(\mathrm{n}=3$ independent experiments; $(*) \mathrm{P} \leq 0.01,(* *) \mathrm{P} \leq 0.001,(* * *) \mathrm{P} \leq 0.0001$. 


\section{TABLES}

Table S1: Tukey's honest significant difference (HSD) multiple pairwise comparison analysis of the rolling duration between different UFI-tagged KG-1a cells.

\begin{tabular}{lllll}
\hline & diff & lwr & upr & adj \\
\hline AF546-AF405 & 6.64 & -14.96 & 28.24 & 0.97 \\
\hline AF680-AF405 & -1.95 & -24.06 & 20.17 & 1.00 \\
\hline AF405/AF546-AF405 & -6.52 & -27.52 & 14.48 & 0.97 \\
\hline AF405/AF680-AF405 & -5.45 & -25.44 & 14.54 & 0.98 \\
\hline AF546/AF680-AF405 & -1.34 & -22.60 & 19.93 & 1.00 \\
\hline Triple-AF405 & -0.35 & -20.52 & 19.82 & 1.00 \\
\hline AF680-AF546 & -8.59 & -30.24 & 13.06 & 0.90 \\
\hline AF405/AF546-AF546 & -13.16 & -33.67 & 7.34 & 0.48 \\
\hline AF405/AF680-AF546 & -12.09 & -31.57 & 7.38 & 0.52 \\
\hline AF546/AF680-AF546 & -7.98 & -28.76 & 12.80 & 0.92 \\
\hline Triple-AF546 & -6.99 & -26.65 & 12.66 & 0.94 \\
\hline AF405/AF546-AF680 & -4.57 & -25.62 & 16.47 & 1.00 \\
\hline AF405/AF680-AF680 & -3.50 & -23.55 & 16.54 & 1.00 \\
\hline AF546/AF680-AF680 & 0.61 & -20.70 & 21.92 & 1.00 \\
\hline Triple-AF680 & 1.60 & -18.62 & 21.82 & 1.00 \\
\hline AF405/AF680-AF405/AF546 & 1.07 & -17.74 & 19.87 & 1.00 \\
\hline AF546/AF680-AF405/AF546 & 5.18 & -14.97 & 25.33 & 0.99 \\
\hline Triple-AF405/AF546 & 6.17 & -12.82 & 25.16 & 0.96 \\
\hline AF546/AF680-AF405/AF680 & 4.11 & -14.99 & 23.22 & 1.00 \\
\hline Triple-AF405/AF680 & 5.10 & -12.77 & 22.98 & 0.98 \\
\hline Triple-AF546/AF680 & 0.99 & -18.30 & 20.27 & 1.00 \\
\hline & & & & \\
\hline
\end{tabular}

diff: difference; lwr: lower; upr: upper; adj: adjusted p-value 
Table S2: Tukey's HSD multiple pairwise comparison analysis of the mean number of rolling cells between different UFI-tagged KG-1a cells.

\begin{tabular}{lllll}
\hline & diff & lwr & upr & adj \\
\hline AF405/AF546-AF405 & 9.33 & -39.86 & 58.52 & 0.99 \\
\hline AF405/AF680-AF405 & 22.33 & -26.86 & 71.52 & 0.71 \\
\hline AF546-AF405 & 3.67 & -45.52 & 52.86 & 1.00 \\
\hline AF546/AF680-AF405 & 6.67 & -42.52 & 55.86 & 1.00 \\
\hline AF680-AF405 & -0.33 & -49.52 & 48.86 & 1.00 \\
\hline Triple-AF405 & 19.67 & -29.52 & 68.86 & 0.81 \\
\hline AF405/AF680-AF405/AF546 & 13.00 & -36.19 & 62.19 & 0.97 \\
\hline AF546-AF405/AF546 & -5.67 & -54.86 & 43.52 & 1.00 \\
\hline AF546/AF680-AF405/AF546 & -2.67 & -51.86 & 46.52 & 1.00 \\
AF680-AF405/AF546 & -9.67 & -58.86 & 39.52 & 0.99 \\
Triple-AF405/AF546 & 10.33 & -38.86 & 59.52 & 0.99 \\
\hline AF546-AF405/AF680 & -18.67 & -67.86 & 30.52 & 0.84 \\
\hline AF546/AF680-AF405/AF680 & -15.67 & -64.86 & 33.52 & 0.92 \\
\hline AF680-AF405/AF680 & -22.67 & -71.86 & 26.52 & 0.70 \\
\hline Triple-AF405/AF680 & -2.67 & -51.86 & 46.52 & 1.00 \\
\hline AF546/AF680-AF546 & 3.00 & -46.19 & 52.19 & 1.00 \\
\hline AF680-AF546 & -4.00 & -53.19 & 45.19 & 1.00 \\
\hline Triple-AF546 & 16.00 & -33.19 & 65.19 & 0.91 \\
\hline AF680-AF546/AF680 & -7.00 & -56.19 & 42.19 & 1.00 \\
\hline Triple-AF546/AF680 & 13.00 & -36.19 & 62.19 & 0.97 \\
\hline Triple-AF680 & 20.00 & -29.19 & 69.19 & 0.80 \\
\hline & & & & \\
\hline & & &
\end{tabular}

diff: difference; lwr: lower; upr: upper; adj: adjusted p-value 
Table S3: Tukey's HSD multiple pairwise comparison analysis of the mean number of rolling cells between different UFI-tagged KG-1a cells at 2 dyne $/ \mathrm{cm}^{2} ;-\mathrm{F}(6 ; 14=0: 673 ; \mathrm{p}=0: 674)$.

\begin{tabular}{lllll}
\hline & diff & lwr & upr & adj \\
\hline AF546-AF405 & 0.11 & -22.26 & 22.47 & 1.00 \\
\hline AF680-AF405 & -5.69 & -28.05 & 16.68 & 0.97 \\
\hline AF405/AF546-AF405 & -1.74 & -24.11 & 20.62 & 1.00 \\
\hline AF405/AF680-AF405 & 1.92 & -20.45 & 24.28 & 1.00 \\
\hline AF546/AF680-AF405 & -2.04 & -24.41 & 20.33 & 1.00 \\
\hline Triple-AF405 & 6.59 & -15.77 & 28.96 & 0.94 \\
\hline AF680-AF546 & -5.80 & -28.16 & 16.57 & 0.97 \\
\hline AF405/AF546-AF546 & -1.85 & -24.22 & 20.51 & 1.00 \\
\hline AF405/AF680-AF546 & 1.81 & -20.56 & 24.18 & 1.00 \\
\hline AF546/AF680-AF546 & -2.15 & -24.51 & 20.22 & 1.00 \\
\hline Triple-AF546 & 6.48 & -15.88 & 28.85 & 0.95 \\
\hline AF405/AF546-AF680 & 3.94 & -18.42 & 26.31 & 1.00 \\
\hline AF405/AF680-AF680 & 7.61 & -14.76 & 29.97 & 0.90 \\
\hline AF546/AF680-AF680 & 3.65 & -18.72 & 26.02 & 1.00 \\
\hline Triple-AF680 & 12.28 & -10.09 & 34.65 & 0.53 \\
\hline AF405/AF680-AF405/AF546 & 3.66 & -18.70 & 26.03 & 1.00 \\
\hline AF546/AF680-AF405/AF546 & -0.29 & -22.66 & 22.07 & 1.00 \\
\hline Triple-AF405/AF546 & 8.34 & -14.03 & 30.70 & 0.85 \\
\hline AF546/AF680-AF405/AF680 & -3.96 & -26.32 & 18.41 & 1.00 \\
\hline Triple-AF405/AF680 & 4.67 & -17.69 & 27.04 & 0.99 \\
\hline Triple-AF546/AF680 & 8.63 & -13.74 & 31.00 & 0.83 \\
\hline & & & \\
& & &
\end{tabular}

diff: difference; lwr: lower; upr: upper; adj: adjusted p-value 
Table S4: Tukey's HSD multiple pairwise comparison analysis of the mean number of rolling cells between different UFI-tagged KG-1a cells at 5 dyne $/ \mathrm{cm}^{2} ;-\mathrm{F}(6 ; 14=1: 205 ; \mathrm{p}=0: 36)$.

\begin{tabular}{lllll}
\hline & diff & lwr & upr & adj \\
\hline AF546-AF405 & 4.06 & -15.76 & 23.87 & 0.99 \\
\hline AF680-AF405 & -3.12 & -22.94 & 16.69 & 1.00 \\
\hline AF405/AF546-AF405 & 4.28 & -15.54 & 24.09 & 0.99 \\
\hline AF405/AF680-AF405 & 7.28 & -12.54 & 27.09 & 0.86 \\
\hline AF546/AF680-AF405 & 0.37 & -19.45 & 20.18 & 1.00 \\
\hline Triple-AF405 & 9.91 & -9.91 & 29.73 & 0.62 \\
\hline AF680-AF546 & -7.18 & -26.99 & 12.64 & 0.87 \\
\hline AF405/AF546-AF546 & 0.22 & -19.59 & 20.04 & 1.00 \\
\hline AF405/AF680-AF546 & 3.22 & -16.59 & 23.04 & 1.00 \\
\hline AF546/AF680-AF546 & -3.69 & -23.51 & 16.13 & 0.99 \\
\hline Triple-AF546 & 5.86 & -13.96 & 25.67 & 0.94 \\
\hline AF405/AF546-AF680 & 7.40 & -12.42 & 27.22 & 0.85 \\
\hline AF405/AF680-AF680 & 10.40 & -9.42 & 30.22 & 0.57 \\
\hline AF546/AF680-AF680 & 3.49 & -16.33 & 23.31 & 1.00 \\
\hline Triple-AF680 & 13.03 & -6.78 & 32.85 & 0.33 \\
\hline AF405/AF680-AF405/AF546 & 3.00 & -16.82 & 22.82 & 1.00 \\
\hline AF546/AF680-AF405/AF546 & -3.91 & -23.73 & 15.91 & 0.99 \\
\hline Triple-AF405/AF546 & 5.63 & -14.18 & 25.45 & 0.95 \\
\hline AF546/AF680-AF405/AF680 & -6.91 & -26.73 & 12.91 & 0.89 \\
\hline Triple-AF405/AF680 & 2.63 & -17.18 & 22.45 & 1.00 \\
\hline Triple-AF546/AF680 & 9.54 & -10.27 & 29.36 & 0.66 \\
\hline & & & & \\
\hline
\end{tabular}

diff: difference; lwr: lower; upr: upper; adj: adjusted p-value 
Table S5: Tukey's HSD multiple pairwise comparison analysis of the mean number of rolling cells between different UFI-tagged KG-1a cells at 7 dyne $/ \mathrm{cm}^{2} ;-\mathrm{F}(6 ; 14=0: 851 ; \mathrm{p}=0: 553)$.

\begin{tabular}{lllll}
\hline & diff & lwr & upr & adj \\
\hline AF546-AF405 & 3.59 & -14.38 & 21.56 & 0.99 \\
AF680-AF405 & -0.86 & -18.82 & 17.11 & 1.00 \\
\hline AF405/AF546-AF405 & 3.42 & -14.54 & 21.39 & 0.99 \\
\hline AF405/AF680-AF405 & 7.83 & -10.13 & 25.80 & 0.75 \\
\hline AF546/AF680-AF405 & 1.88 & -16.09 & 19.84 & 1.00 \\
\hline Triple-AF405 & 7.76 & -10.21 & 25.72 & 0.75 \\
\hline AF680-AF546 & -4.44 & -22.41 & 13.52 & 0.98 \\
\hline AF405/AF546-AF546 & -0.17 & -18.13 & 17.80 & 1.00 \\
\hline AF405/AF680-AF546 & 4.24 & -13.72 & 22.21 & 0.98 \\
AF546/AF680-AF546 & -1.71 & -19.68 & 16.26 & 1.00 \\
\hline Triple-AF546 & 4.17 & -13.80 & 22.13 & 0.98 \\
AF405/AF546-AF680 & 4.28 & -13.69 & 22.24 & 0.98 \\
\hline AF405/AF680-AF680 & 8.69 & -9.28 & 26.66 & 0.66 \\
\hline AF546/AF680-AF680 & 2.73 & -15.23 & 20.70 & 1.00 \\
\hline Triple-AF680 & 8.61 & -9.36 & 26.58 & 0.66 \\
\hline AF405/AF680-AF405/AF546 & 4.41 & -13.56 & 22.38 & 0.98 \\
\hline AF546/AF680-AF405/AF546 & -1.54 & -19.51 & 16.42 & 1.00 \\
\hline Triple-AF405/AF546 & 4.33 & -13.63 & 22.30 & 0.98 \\
\hline AF546/AF680-AF405/AF680 & -5.96 & -23.92 & 12.01 & 0.91 \\
\hline Triple-AF405/AF680 & -0.08 & -18.04 & 17.89 & 1.00 \\
\hline Triple-AF546/AF680 & 5.88 & -12.09 & 23.84 & 0.91 \\
\hline
\end{tabular}

diff: difference; lwr: lower; upr: upper; adj: adjusted p-value 
Table S6: Tukey's HSD multiple pairwise comparison analysis of the mean number of rolling cells between different UFI-tagged KG-1a cells at 10 dyne $/ \mathrm{cm}^{2} ;-\mathrm{F}(6 ; 14=0: 358 ; \mathrm{p}=0: 893)$.

\begin{tabular}{lllll}
\hline & diff & lwr & upr & adj \\
\hline AF546-AF405 - & 0.53 & -17.32 & 16.25 & 1.00 \\
\hline AF680-AF405 & -0.91 & -17.69 & 15.87 & 1.00 \\
\hline AF405/AF546-AF405 & -0.92 & -17.70 & 15.87 & 1.00 \\
\hline AF405/AF680-AF405 & 4.87 & -11.91 & 21.65 & 0.95 \\
\hline AF546/AF680-AF405 & -0.54 & -17.32 & 16.24 & 1.00 \\
\hline Triple-AF405 & 1.21 & -15.57 & 17.99 & 1.00 \\
\hline AF680-AF546 & -0.38 & -17.16 & 16.41 & 1.00 \\
\hline AF405/AF546-AF546 & -0.38 & -17.17 & 16.40 & 1.00 \\
\hline AF405/AF680-AF546 & 5.40 & -11.38 & 22.18 & 0.92 \\
\hline AF546/AF680-AF546 & -0.01 & -16.79 & 16.78 & 1.00 \\
\hline Triple-AF546 & 1.74 & -15.04 & 18.53 & 1.00 \\
\hline AF405/AF546-AF680 & -0.01 & -16.79 & 16.78 & 1.00 \\
\hline AF405/AF680-AF680 & 5.78 & -11.01 & 22.56 & 0.89 \\
\hline AF546/AF680-AF680 & 0.37 & -16.41 & 17.15 & 1.00 \\
\hline Triple-AF680 & 2.12 & -14.66 & 18.90 & 1.00 \\
\hline AF405/AF680-AF405/AF546 & 5.78 & -11.00 & 22.57 & 0.89 \\
\hline AF546/AF680-AF405/AF546 & 0.38 & -16.41 & 17.16 & 1.00 \\
\hline Triple-AF405/AF546 & 2.13 & -14.66 & 18.91 & 1.00 \\
\hline AF546/AF680-AF405/AF680 & -5.41 & -22.19 & 11.37 & 0.92 \\
\hline Triple-AF405/AF680 & -3.66 & -20.44 & 13.12 & 0.99 \\
\hline Triple-AF546/AF680 & 1.75 & -15.03 & 18.53 & 1.00 \\
\hline & & & & \\
\hline
\end{tabular}

diff: difference; lwr: lower; upr: upper; adj: adjusted p-value 
Table S7: Tukey's HSD multiple pairwise comparison analysis of the mean number of rolling cells between different UFI-tagged KG-1a cells at 15 dyne/ $\mathrm{cm}^{2} ;-\mathrm{F}(6 ; 14=0: 454 ; \mathrm{p}=0: 831)$.

\begin{tabular}{lllll}
\hline & diff & lwr & upr & adj \\
\hline AF546-AF405 & 0.44 & -14.54 & 15.43 & 1.00 \\
& & & & \\
\hline AF680-AF405 & -0.38 & -15.37 & 14.60 & 1.00 \\
\hline AF405/AF546-AF405 & -1.92 & -16.90 & 13.07 & 1.00 \\
\hline AF405/AF680-AF405 & 4.72 & -10.26 & 19.71 & 0.93 \\
\hline AF546/AF680-AF405 & 1.34 & -13.65 & 16.32 & 1.00 \\
\hline Triple-AF405 & 1.81 & -13.17 & 16.80 & 1.00 \\
\hline AF680-AF546 & -0.82 & -15.81 & 14.16 & 1.00 \\
\hline AF405/AF546-AF546 & -2.36 & -17.35 & 12.62 & 1.00 \\
\hline AF405/AF680-AF546 & 4.28 & -10.71 & 19.26 & 0.95 \\
AF546/AF680-AF546 & 0.89 & -14.09 & 15.88 & 1.00 \\
Triple-AF546 & 1.37 & -13.61 & 16.36 & 1.00 \\
\hline AF405/AF546-AF680 & -1.54 & -16.52 & 13.45 & 1.00 \\
\hline AF405/AF680-AF680 & 5.10 & -9.88 & 20.09 & 0.90 \\
\hline AF546/AF680-AF680 & 1.72 & -13.27 & 16.70 & 1.00 \\
\hline Triple-AF680 & 2.20 & -12.79 & 17.18 & 1.00 \\
\hline AF405/AF680-AF405/AF546 & 6.64 & -8.34 & 21.63 & 0.73 \\
\hline AF546/AF680-AF405/AF546 & 3.26 & -11.73 & 18.24 & 0.99 \\
\hline Triple-AF405/AF546 & 3.73 & -11.25 & 18.72 & 0.97 \\
\hline AF546/AF680-AF405/AF680 & -3.38 & -18.37 & 11.60 & 0.98 \\
\hline Triple-AF405/AF680 & -2.91 & -17.89 & 12.08 & 0.99 \\
\hline Triple-AF546/AF680 & 0.48 & -14.51 & 15.46 & 1.00 \\
\hline
\end{tabular}

diff: difference; lwr: lower; upr: upper; adj: adjusted p-value 
Table S8: Tukey's HSD multiple pairwise comparison analysis of the mean number of rolling cells between different UFI-tagged KG-1a cells at 20 dyne/ $\mathrm{cm}^{2} ;-\mathrm{F}(6 ; 14=1: 258 ; \mathrm{p}=0: 337)$.

\begin{tabular}{lllll}
\hline & diff & lwr & upr & adj \\
\hline AF546-AF405 & 2.63 & -12.02 & 17.29 & 1.00 \\
AF680-AF405 & 1.26 & -13.40 & 15.91 & 1.00 \\
\hline AF405/AF546-AF405 & 1.00 & -13.66 & 15.66 & 1.00 \\
\hline AF405/AF680-AF405 & 9.18 & -5.48 & 23.84 & 0.38 \\
\hline AF546/AF680-AF405 & 4.41 & -10.25 & 19.07 & 0.94 \\
\hline Triple-AF405 & 7.01 & -7.65 & 21.67 & 0.67 \\
\hline AF680-AF546 & -1.38 & -16.04 & 13.28 & 1.00 \\
\hline AF405/AF546-AF546 & -1.63 & -16.29 & 13.02 & 1.00 \\
\hline AF405/AF680-AF546 & 6.54 & -8.11 & 21.20 & 0.73 \\
AF546/AF680-AF546 & 1.78 & -12.88 & 16.44 & 1.00 \\
\hline Triple-AF546 & 4.38 & -10.28 & 19.04 & 0.94 \\
AF405/AF546-AF680 & -0.26 & -14.91 & 14.40 & 1.00 \\
\hline AF405/AF680-AF680 & 7.92 & -6.74 & 22.58 & 0.54 \\
AF546/AF680-AF680 & 3.16 & -11.50 & 17.81 & 0.99 \\
\hline Triple-AF680 & 5.76 & -8.90 & 20.41 & 0.82 \\
\hline AF405/AF680-AF405/AF546 & 8.18 & -6.48 & 22.84 & 0.51 \\
\hline AF546/AF680-AF405/AF546 & 3.41 & -11.25 & 18.07 & 0.98 \\
\hline Triple-AF405/AF546 & 6.01 & -8.65 & 20.67 & 0.79 \\
\hline AF546/AF680-AF405/AF680 & -4.77 & -19.42 & 9.89 & 0.91 \\
\hline Triple-AF405/AF680 & -2.17 & -16.82 & 12.49 & 1.00 \\
\hline Triple-AF546/AF680 & 2.60 & -12.06 & 17.26 & 1.00 \\
\hline
\end{tabular}

diff: difference; lwr: lower; upr: upper; adj: adjusted p-value 
Table S9: Tukey's HSD multiple pairwise comparison analysis of the velocity of rolling cells between different UFI-tagged KG-1a cells at 2 dyne $/ \mathrm{cm}^{2} ;-\mathrm{F}(6 ; 14=0: 575 ; \mathrm{p}=0: 744)$.

\begin{tabular}{lllll}
\hline & diff & lwr & upr & adj \\
\hline AF546-AF405 & -0.37 & -1.44 & 0.69 & 0.88 \\
\hline AF680-AF405 & -0.40 & -1.46 & 0.67 & 0.85 \\
\hline AF405/AF546-AF405 & -0.51 & -1.57 & 0.56 & 0.67 \\
\hline AF405/AF680-AF405 & -0.23 & -1.29 & 0.84 & 0.99 \\
\hline AF546/AF680-AF405 & -0.20 & -1.26 & 0.87 & 0.99 \\
\hline Triple-AF405 & -0.37 & -1.44 & 0.69 & 0.88 \\
\hline AF680-AF546 & -0.02 & -1.09 & 1.04 & 1.00 \\
\hline AF405/AF546-AF546 & -0.13 & -1.20 & 0.93 & 1.00 \\
\hline AF405/AF680-AF546 & 0.14 & -0.92 & 1.21 & 1.00 \\
\hline AF546/AF680-AF546 & 0.18 & -0.89 & 1.24 & 1.00 \\
\hline Triple-AF546 & 0.00 & -1.06 & 1.07 & 1.00 \\
\hline AF405/AF546-AF680 & -0.11 & -1.17 & 0.95 & 1.00 \\
\hline AF405/AF680-AF680 & 0.17 & -0.90 & 1.23 & 1.00 \\
\hline AF546/AF680-AF680 & 0.20 & -0.87 & 1.26 & 0.99 \\
\hline Triple-AF680 & 0.02 & -1.04 & 1.09 & 1.00 \\
\hline AF405/AF680-AF405/AF546 & 0.28 & -0.79 & 1.34 & 0.97 \\
\hline AF546/AF680-AF405/AF546 & 0.31 & -0.76 & 1.37 & 0.95 \\
\hline Triple-AF405/AF546 & 0.13 & -0.93 & 1.20 & 1.00 \\
\hline AF546/AF680-AF405/AF680 & 0.03 & -1.03 & 1.10 & 1.00 \\
\hline Triple-AF405/AF680 & -0.14 & -1.21 & 0.92 & 1.00 \\
\hline Triple-AF546/AF680 & -0.18 & -1.24 & 0.89 & 1.00 \\
\hline
\end{tabular}

diff: difference; lwr: lower; upr: upper; adj: adjusted p-value 
Table S10: Tukey's HSD multiple pairwise comparison analysis of the velocity of rolling cells between different UFI-tagged KG-1a cells at 5 dyne $/ \mathrm{cm}^{2} ;-\mathrm{F}(6 ; 14=0: 796 ; \mathrm{p}=0: 588)$.

\begin{tabular}{|c|c|c|c|c|}
\hline & diff & lwr & upr & adj \\
\hline AF546-AF405 & -0.24 & -1.19 & 0.70 & 0.97 \\
\hline AF680-AF405 & 0.05 & -0.89 & 0.99 & 1.00 \\
\hline AF405/AF546-AF405 & -0.15 & -1.10 & 0.79 & 1.00 \\
\hline AF405/AF680-AF405 & -0.18 & -1.12 & 0.77 & 0.99 \\
\hline AF546/AF680-AF405 & -0.48 & -1.43 & 0.46 & 0.60 \\
\hline Triple-AF405 & -0.21 & -1.15 & 0.74 & 0.99 \\
\hline AF680-AF546 & 0.29 & -0.65 & 1.24 & 0.93 \\
\hline AF405/AF546-AF546 & 0.09 & -0.85 & 1.04 & 1.00 \\
\hline AF405/AF680-AF546 & 0.06 & -0.88 & 1.01 & 1.00 \\
\hline AF546/AF680-AF546 & -0.24 & -1.19 & 0.70 & 0.97 \\
\hline Triple-AF546 & 0.03 & -0.91 & 0.98 & 1.00 \\
\hline AF405/AF546-AF680 & -0.20 & -1.15 & 0.74 & 0.99 \\
\hline AF405/AF680-AF680 & -0.23 & -1.17 & 0.72 & 0.98 \\
\hline AF546/AF680-AF680 & -0.53 & -1.48 & 0.41 & 0.49 \\
\hline Triple-AF680 & -0.26 & -1.20 & 0.69 & 0.96 \\
\hline AF405/AF680-AF405/AF546 & -0.03 & -0.97 & 0.92 & 1.00 \\
\hline AF546/AF680-AF405/AF546 & -0.33 & -1.28 & 0.61 & 0.88 \\
\hline Triple-AF405/AF546 & -0.06 & -1.00 & 0.89 & 1.00 \\
\hline AF546/AF680-AF405/AF680 & -0.31 & -1.25 & 0.64 & 0.92 \\
\hline Triple-AF405/AF680 & -0.03 & -0.97 & 0.92 & 1.00 \\
\hline Triple-AF546/AF680 & 0.28 & -0.67 & 1.22 & 0.95 \\
\hline
\end{tabular}

diff: difference; lwr: lower; upr: upper; adj: adjusted p-value 
Table S11: Tukey's HSD multiple pairwise comparison analysis of the velocity of rolling cells between different UFI-tagged KG-1a cells at 7 dyne/ $\mathrm{cm}^{2} ;-\mathrm{F}(6 ; 14=3: 296 ; \mathrm{p}=0: 0309)$.

\begin{tabular}{lllll}
\hline & Diff & lwr & upr & adj \\
\hline AF546-AF405 & 0.01 & -0.45 & 0.46 & 1.00 \\
AF680-AF405 & 0.16 & -0.30 & 0.62 & 0.88 \\
\hline AF405/AF546-AF405 & -0.03 & -0.49 & 0.42 & 1.00 \\
\hline AF405/AF680-AF405 & -0.04 & -0.50 & 0.42 & 1.00 \\
\hline AF546/AF680-AF405 & -0.38 & -0.84 & 0.08 & 0.14 \\
\hline Triple-AF405 & -0.19 & -0.65 & 0.26 & 0.78 \\
\hline AF680-AF546 & 0.16 & -0.30 & 0.61 & 0.90 \\
\hline AF405/AF546-AF546 & -0.04 & -0.50 & 0.42 & 1.00 \\
\hline AF405/AF680-AF546 & -0.05 & -0.50 & 0.41 & 1.00 \\
\hline AF546/AF680-AF546 & -0.39 & -0.84 & 0.07 & 0.13 \\
\hline Triple-AF546 & -0.20 & -0.65 & 0.26 & 0.75 \\
AF405/AF546-AF680 & -0.20 & -0.65 & 0.26 & 0.76 \\
\hline AF405/AF680-AF680 & -0.20 & -0.66 & 0.25 & 0.73 \\
\hline AF546/AF680-AF680 & -0.54 & -1.00 & -0.08 & 0.02 \\
\hline Triple-AF680 & -0.35 & -0.81 & 0.10 & 0.19 \\
\hline AF405/AF680-AF405/AF546 & -0.01 & -0.46 & 0.45 & 1.00 \\
\hline AF546/AF680-AF405/AF546 & -0.35 & -0.80 & 0.11 & 0.20 \\
\hline Triple-AF405/AF546 & -0.16 & -0.61 & 0.30 & 0.89 \\
\hline AF546/AF680-AF405/AF680 & -0.34 & -0.79 & 0.12 & 0.22 \\
\hline Triple-AF405/AF680 & -0.15 & -0.61 & 0.31 & 0.91 \\
\hline Triple-AF546/AF680 & 0.19 & -0.27 & 0.64 & 0.79 \\
\hline
\end{tabular}

diff: difference; lwr: lower; upr: upper; adj: adjusted p-value 
Table S12: Tukey's HSD multiple pairwise comparison analysis of the velocity of rolling cells between different UFI-tagged KG-1a cells at 10 dyne $/ \mathrm{cm}^{2} ;-F(6 ; 14=3: 421 ; \mathrm{p}=0: 0271)$.

\begin{tabular}{lllll}
\hline & diff & lwr & upr & adj \\
\hline AF546-AF405 & 0.40 & -0.12 & 0.91 & 0.19 \\
AF680-AF405 & 0.22 & -0.29 & 0.74 & 0.76 \\
\hline AF405/AF546-AF405 & 0.15 & -0.36 & 0.67 & 0.94 \\
\hline AF405/AF680-AF405 & -0.07 & -0.58 & 0.45 & 1.00 \\
\hline AF546/AF680-AF405 & -0.08 & -0.59 & 0.44 & 1.00 \\
\hline Triple-AF405 & -0.16 & -0.68 & 0.36 & 0.93 \\
\hline AF680-AF546 & -0.17 & -0.69 & 0.34 & 0.90 \\
\hline AF405/AF546-AF546 & -0.24 & -0.76 & 0.27 & 0.68 \\
\hline AF405/AF680-AF546 & -0.46 & -0.98 & 0.05 & 0.09 \\
\hline AF546/AF680-AF546 & -0.47 & -0.99 & 0.04 & 0.08 \\
\hline Triple-AF546 & -0.56 & -1.07 & -0.04 & 0.03 \\
AF405/AF546-AF680 & -0.07 & -0.58 & 0.45 & 1.00 \\
\hline AF405/AF680-AF680 & -0.29 & -0.81 & 0.23 & 0.50 \\
\hline AF546/AF680-AF680 & -0.30 & -0.81 & 0.22 & 0.47 \\
\hline Triple-AF680 & -0.38 & -0.90 & 0.13 & 0.22 \\
\hline AF405/AF680-AF405/AF546 & -0.22 & -0.74 & 0.30 & 0.76 \\
\hline AF546/AF680-AF405/AF546 & -0.23 & -0.75 & 0.29 & 0.73 \\
\hline Triple-AF405/AF546 & -0.31 & -0.83 & 0.20 & 0.42 \\
\hline AF546/AF680-AF405/AF680 & -0.01 & -0.52 & 0.51 & 1.00 \\
\hline Triple-AF405/AF680 & -0.09 & -0.61 & 0.42 & 1.00 \\
\hline Triple-AF546/AF680 & -0.08 & -0.60 & 0.43 & 1.00 \\
\hline
\end{tabular}

diff: difference; lwr: lower; upr: upper; adj: adjusted p-value 
Table S13: Tukey's HSD multiple pairwise comparison analysis of the velocity of rolling cells between different UFI-tagged KG-1a cells at 15 dyne $/ \mathrm{cm}^{2} ;-\mathrm{F}(6 ; 14=1: 231 ; \mathrm{p}=0: 348)$.

\begin{tabular}{|c|c|c|c|c|}
\hline & diff & lwr & upr & adj \\
\hline AF546-AF405 & 0.19 & -0.72 & 1.09 & 0.99 \\
\hline AF680-AF405 & 0.09 & -0.81 & 1.00 & 1.00 \\
\hline AF405/AF546-AF405 & -0.19 & -1.10 & 0.72 & 0.99 \\
\hline AF405/AF680-AF405 & -0.24 & -1.15 & 0.66 & 0.96 \\
\hline AF546/AF680-AF405 & -0.42 & -1.33 & 0.49 & 0.70 \\
\hline Triple-AF405 & -0.11 & -1.02 & 0.79 & 1.00 \\
\hline AF680-AF546 & -0.09 & -1.00 & 0.82 & 1.00 \\
\hline AF405/AF546-AF546 & -0.38 & -1.29 & 0.53 & 0.78 \\
\hline AF405/AF680-AF546 & -0.43 & -1.34 & 0.48 & 0.68 \\
\hline AF546/AF680-AF546 & -0.61 & -1.51 & 0.30 & 0.32 \\
\hline Triple-AF546 & -0.30 & -1.21 & 0.61 & 0.91 \\
\hline AF405/AF546-AF680 & -0.29 & -1.20 & 0.62 & 0.92 \\
\hline AF405/AF680-AF680 & -0.34 & -1.25 & 0.57 & 0.85 \\
\hline AF546/AF680-AF680 & -0.51 & -1.42 & 0.39 & 0.49 \\
\hline Triple-AF680 & -0.21 & -1.12 & 0.70 & 0.98 \\
\hline AF405/AF680-AF405/AF546 & -0.05 & -0.96 & 0.86 & 1.00 \\
\hline AF546/AF680-AF405/AF546 & -0.23 & -1.14 & 0.68 & 0.97 \\
\hline Triple-AF405/AF546 & 0.08 & -0.83 & 0.99 & 1.00 \\
\hline AF546/AF680-AF405/AF680 & -0.18 & -1.08 & 0.73 & 0.99 \\
\hline Triple-AF405/AF680 & 0.13 & -0.78 & 1.04 & 1.00 \\
\hline Triple-AF546/AF680 & 0.31 & -0.60 & 1.21 & 0.90 \\
\hline
\end{tabular}

diff: difference; lwr: lower; upr: upper; adj: adjusted p-value 
Table S14: Tukey's HSD multiple pairwise comparison analysis of the velocity of rolling cells between different UFI-tagged KG-1a cells at 20 dyne/ $\mathrm{cm}^{2} ;-\mathrm{F}(6 ; 14=1: 399 ; \mathrm{p}=0: 282)$.

\begin{tabular}{|c|c|c|c|c|}
\hline & diff & lwr & upr & adj \\
\hline AF546-AF405 & 0.54 & -0.57 & 1.64 & 0.65 \\
\hline AF680-AF405 & -0.16 & -1.26 & 0.95 & 1.00 \\
\hline AF405/AF546-AF405 & -0.30 & -1.41 & 0.80 & 0.96 \\
\hline AF405/AF680-AF405 & -0.04 & -1.15 & 1.06 & 1.00 \\
\hline AF546/AF680-AF405 & -0.08 & -1.19 & 1.02 & 1.00 \\
\hline Triple-AF405 & -0.18 & -1.29 & 0.92 & 1.00 \\
\hline AF680-AF546 & -0.69 & -1.80 & 0.41 & 0.38 \\
\hline AF405/AF546-AF546 & -0.84 & -1.94 & 0.26 & 0.20 \\
\hline AF405/AF680-AF546 & -0.58 & -1.68 & 0.52 & 0.57 \\
\hline AF546/AF680-AF546 & -0.62 & -1.72 & 0.48 & 0.50 \\
\hline Triple-AF546 & -0.72 & -1.82 & 0.39 & 0.34 \\
\hline AF405/AF546-AF680 & -0.15 & -1.25 & 0.96 & 1.00 \\
\hline AF405/AF680-AF680 & 0.11 & -0.99 & 1.22 & 1.00 \\
\hline AF546/AF680-AF680 & 0.07 & -1.03 & 1.18 & 1.00 \\
\hline Triple-AF680 & -0.02 & -1.13 & 1.08 & 1.00 \\
\hline AF405/AF680-AF405/AF546 & 0.26 & -0.84 & 1.36 & 0.98 \\
\hline AF546/AF680-AF405/AF546 & 0.22 & -0.88 & 1.32 & 0.99 \\
\hline Triple-AF405/AF546 & 0.12 & -0.98 & 1.22 & 1.00 \\
\hline AF546/AF680-AF405/AF680 & -0.04 & -1.14 & 1.06 & 1.00 \\
\hline Triple-AF405/AF680 & -0.14 & -1.24 & 0.97 & 1.00 \\
\hline Triple-AF546/AF680 & -0.10 & -1.20 & 1.01 & 1.00 \\
\hline
\end{tabular}

diff: difference; lwr: lower; upr: upper; adj: adjusted p-value 
FMCR SAMPLE ANALYSIS PIPELINE: 


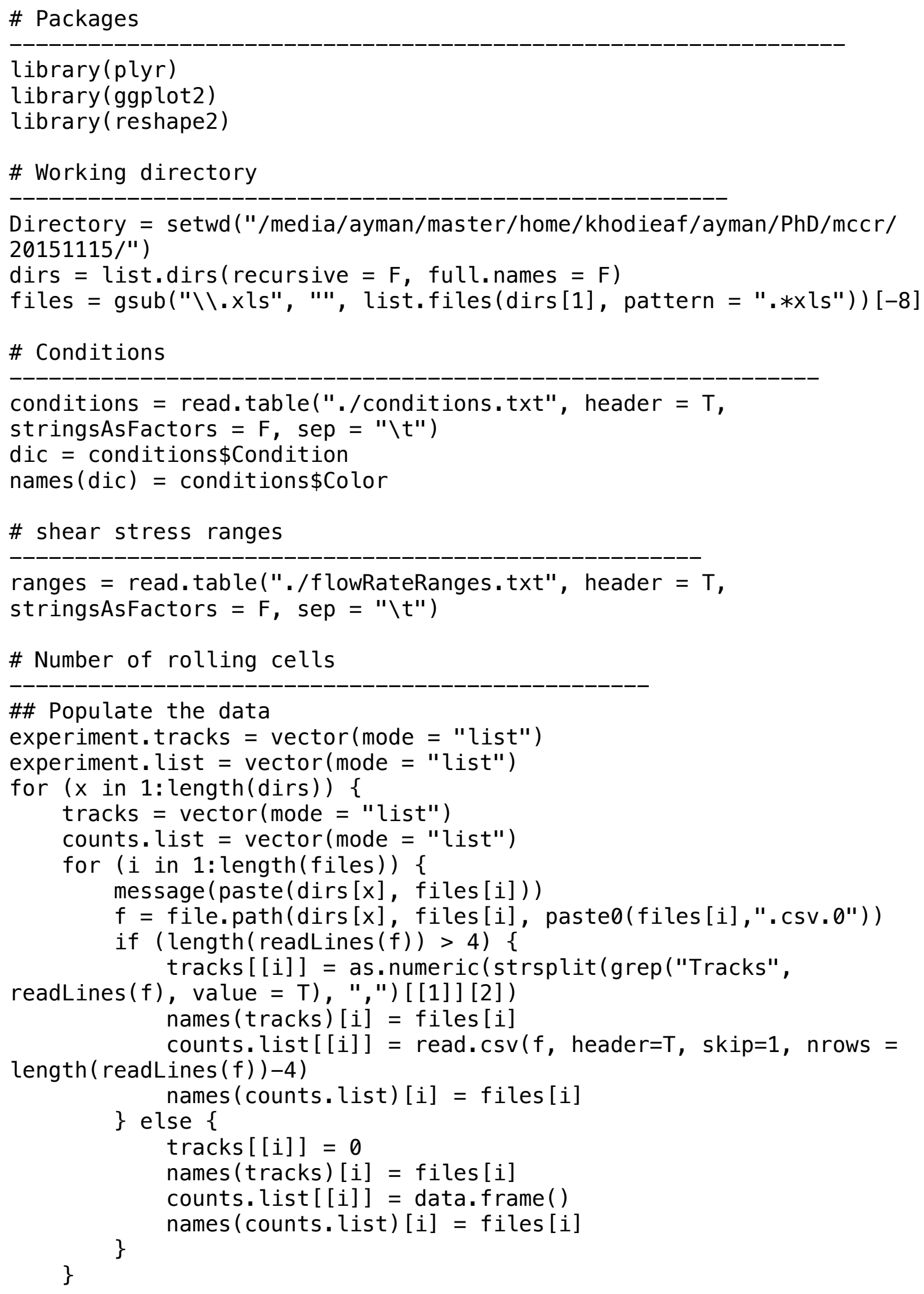




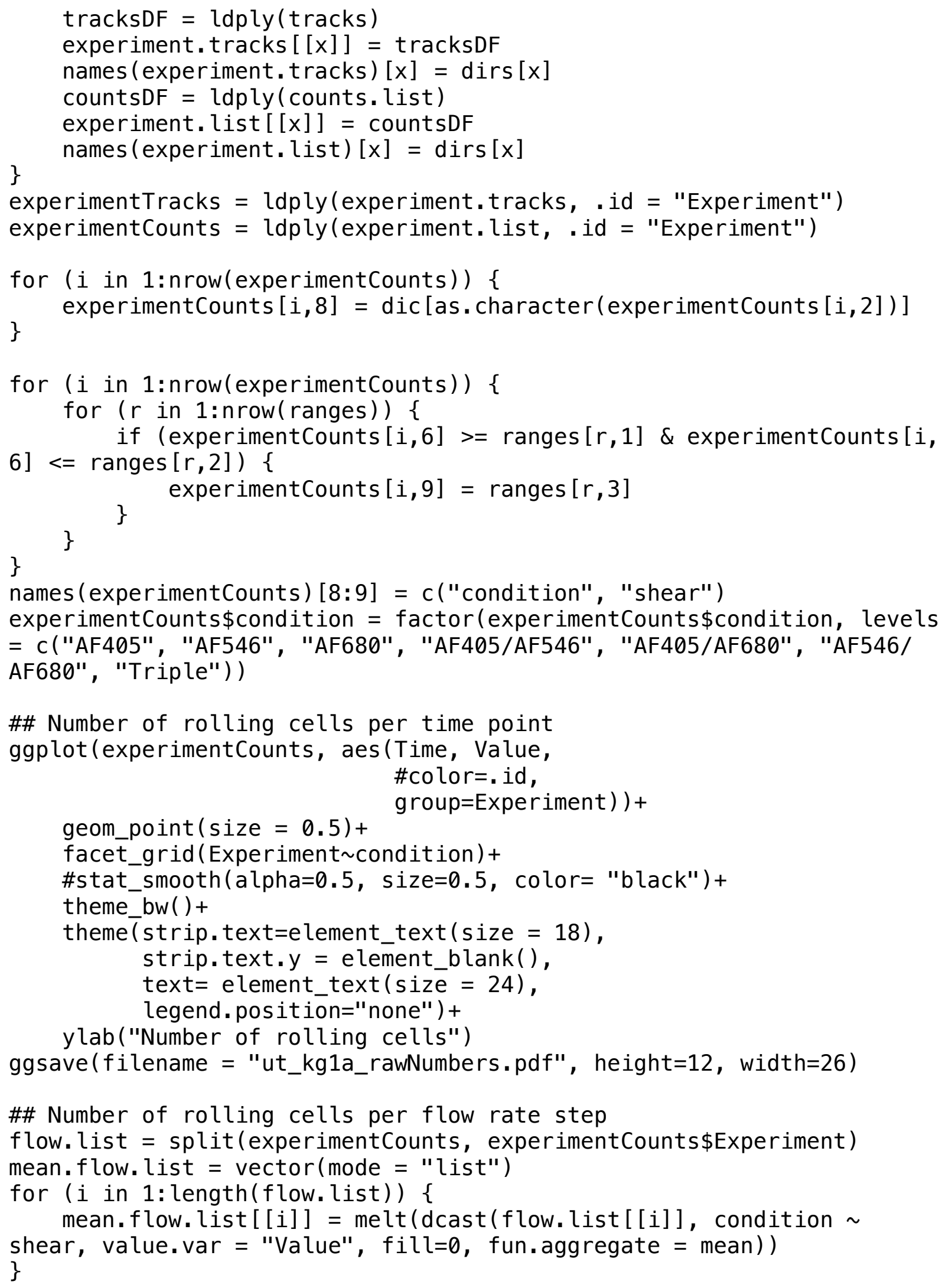


mean.flow. DF = cbind (mean.flow. list [ [1]], mean.flow. list [ [2] ]\$value, mean. flow. list [ [3] ] \$value)

mean.flow.DF\$mean = apply (mean.flow.DF $[, 3: 5], 1$, mean $)$

mean.flow.DF\$sd = apply (mean.flow.DF $[, 3: 5], 1, \mathrm{sd})$

mean. flow. DF\$variable $=$

as. numeric (as. character (mean. flow. DF\$variable))

mean.flow. DF\$condition = factor(mean.flow.DF\$condition, levels = c("AF405", "AF546", "AF680", "AF405/AF546", "AF405/AF680", "AF546/ AF680", "Triple"))

ggplot(mean.flow.DF, aes(variable, mean, \#shape=condition,

geom_point $($ size $=1.5)+$ group=condition) ) +

geom_line(aes(variable,mean), size=0.5)+

\#scale_color_manual (values = c("black", "blue", "darkgreen",

"red", "cyan", "purple", "orange", "pink"), name="Condition")+ geom_errorbar (aes (ymax=mean+sd, ymin=mean-sd), width=0.1)+ theme_bw ()$+$

theme (text=element_text $($ size $=16))+$

xlab(expression(paste("Shear stress (dyne/cm"^"2",")")))+

ylab("Number of rolling cells")+

scale_linetype_discrete(name="Condition") +

scale_shape_discrete $($ name="Condition" $)+$

facet_grid(. condition)+

\#theme (legend.position="none" )+

$y \lim (c(0,40))$

ggsave("ut_kgla_NumbersPerShear.pdf", height=3, width=18)

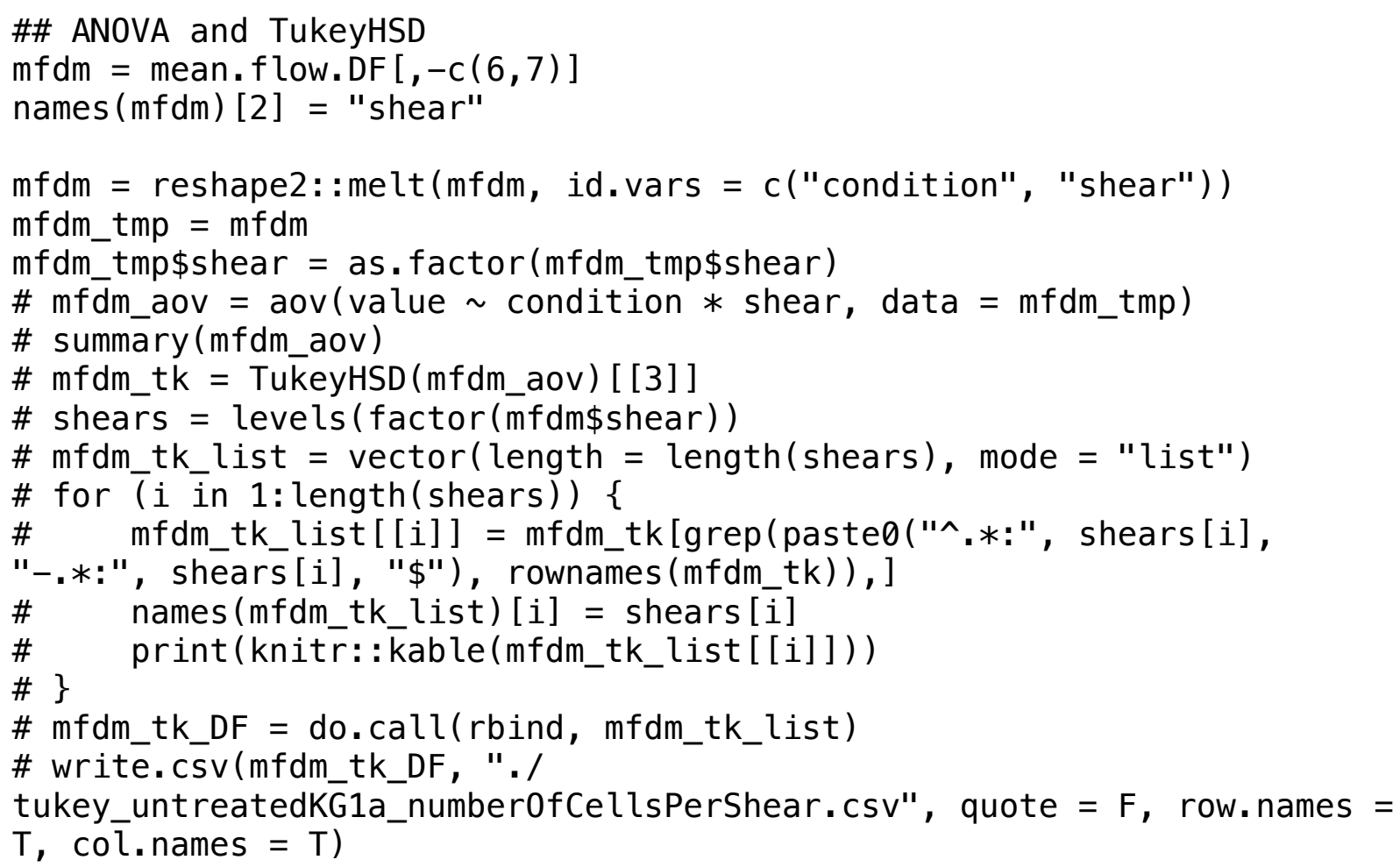




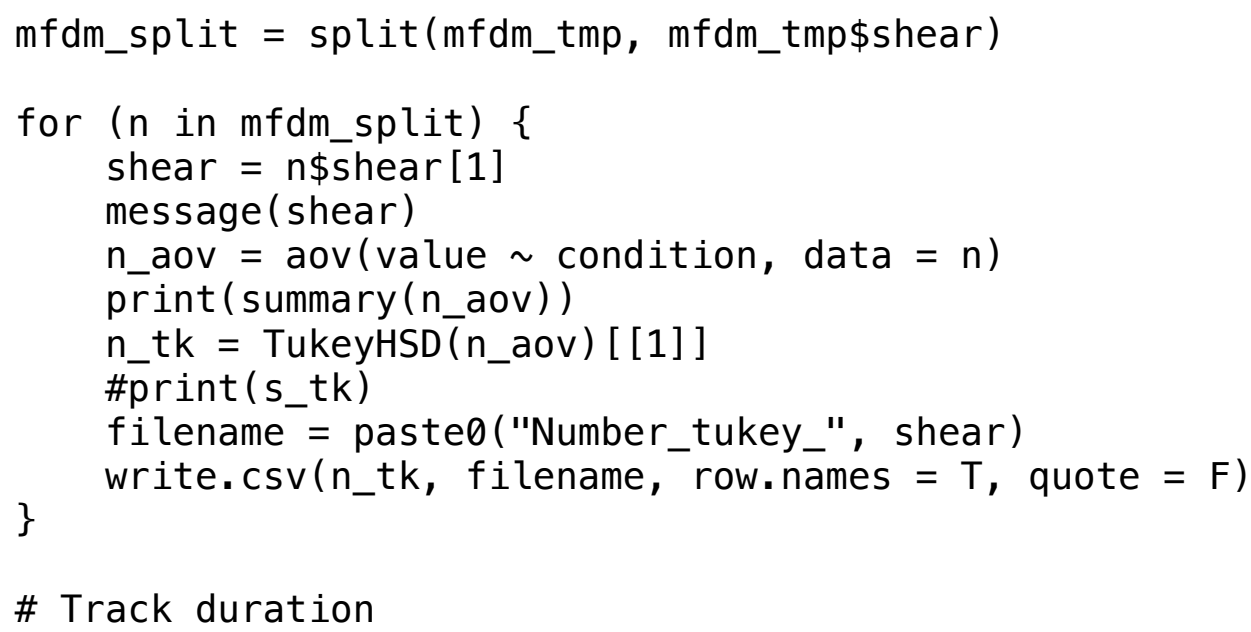




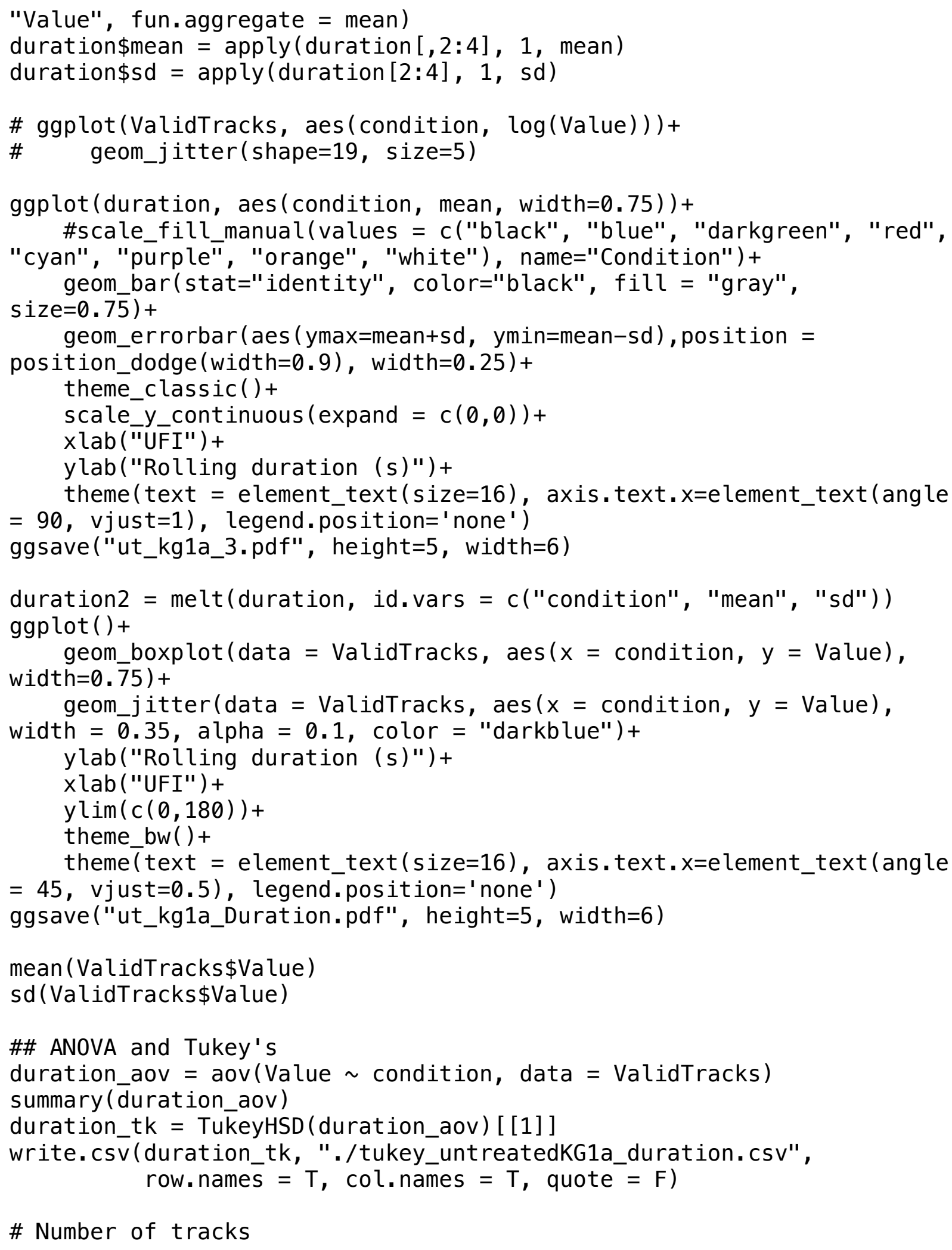


tracks\$color $=$ as. character (tracks\$color)

for ( $i$ in 1:nrow(tracks)) \{tracks $[i, 4]=\operatorname{dic}[\operatorname{tracks}[i, 2]]\}$

names(tracks) $[4]=$ "condition"

\#\# find the mean of 2 replicates

meanTracks = dcast (tracks, condition $\sim$ experiment , value.var =

"number.of.cells", fun.aggregate = mean)

meanTracks\$mean $=\operatorname{apply}($ meanTracks $[, 2: 4], 1$, mean $)$

meanTracks $\$ s d=\operatorname{apply}($ meanTracks $[, 2: 4], 1, s d)$

meanTracks\$condition = factor $($ meanTracks\$condition, levels =

c("AF405", "AF546", "AF680", "AF405/AF546", "AF405/AF680", "AF546/

AF680", "Triple"))

ggplot (meanTracks, aes(condition, mean, width=0.75)) + geom_bar(stat="identity", fill="white", color="black", size=0.5)+ geom_errorbar (aes (ymax=mean+sd, ymin=mean-sd), position =

position_dodge $($ width $=0.9)$, width $=0.25)+$

theme_classic( )+

scale_y_continuous (expand $=c(0,0))+$

xlab ("Treatment") +

ylab("Total number of rolling cells")+

$=90)$ )

theme $($ text $=$ element_text $(\operatorname{size}=16)$, axis.text.x=element_text (angle

ggsave("ut_kg1a_TotalNumber.pdf", height=5, width=6)

mean (as. vector (as.matrix(meanTracks [, c(2:4)])))

sd(as. vector (as.matrix (meanTracks [, c(2:4)])))

\#\# ANOVA and Tukey's

tracks_aov $=$ aov (number. of. cells $\sim$ condition, data $=$ tracks $)$

summary(tracks_aov)

tracks_tk = TukeyHSD (tracks_aov) [ [1] ]

write.csv(tracks_tk, "./tukey_untreatedKGla_number0fCellsTotal.csv",

row. names $=T$, col. names $=T$, quote $=F$ )

\# Track displacement

\#\# Populate data

TrackDisplacement. list $=\operatorname{vector}($ mode $=$ "list" $)$

for ( $x$ in 1: length(dirs)) \{

trackDisplacement. list $=\operatorname{vector}($ mode $=$ "list")

for ( $i$ in 1: length (files)) \{ message (paste(dirs $[x]$, files $[i]))$

$f=$ file.path(dirs $[x]$, files $[i], \operatorname{paste0}(f i l e s[i], " . c s v .43 ")$ )

if ( $f \%$ in\% list.files(file.path(dirs $[x]$, files[i]), full.names

$=\mathrm{T}))\{$

if (length (readLines $(f))>3$ ) \{

trackDisplacement. list $[i]]=\operatorname{read} . \operatorname{csv}(f$, header $=T$,

skip=1)

names(trackDisplacement. list) $[i]=$ files $[i]$ 


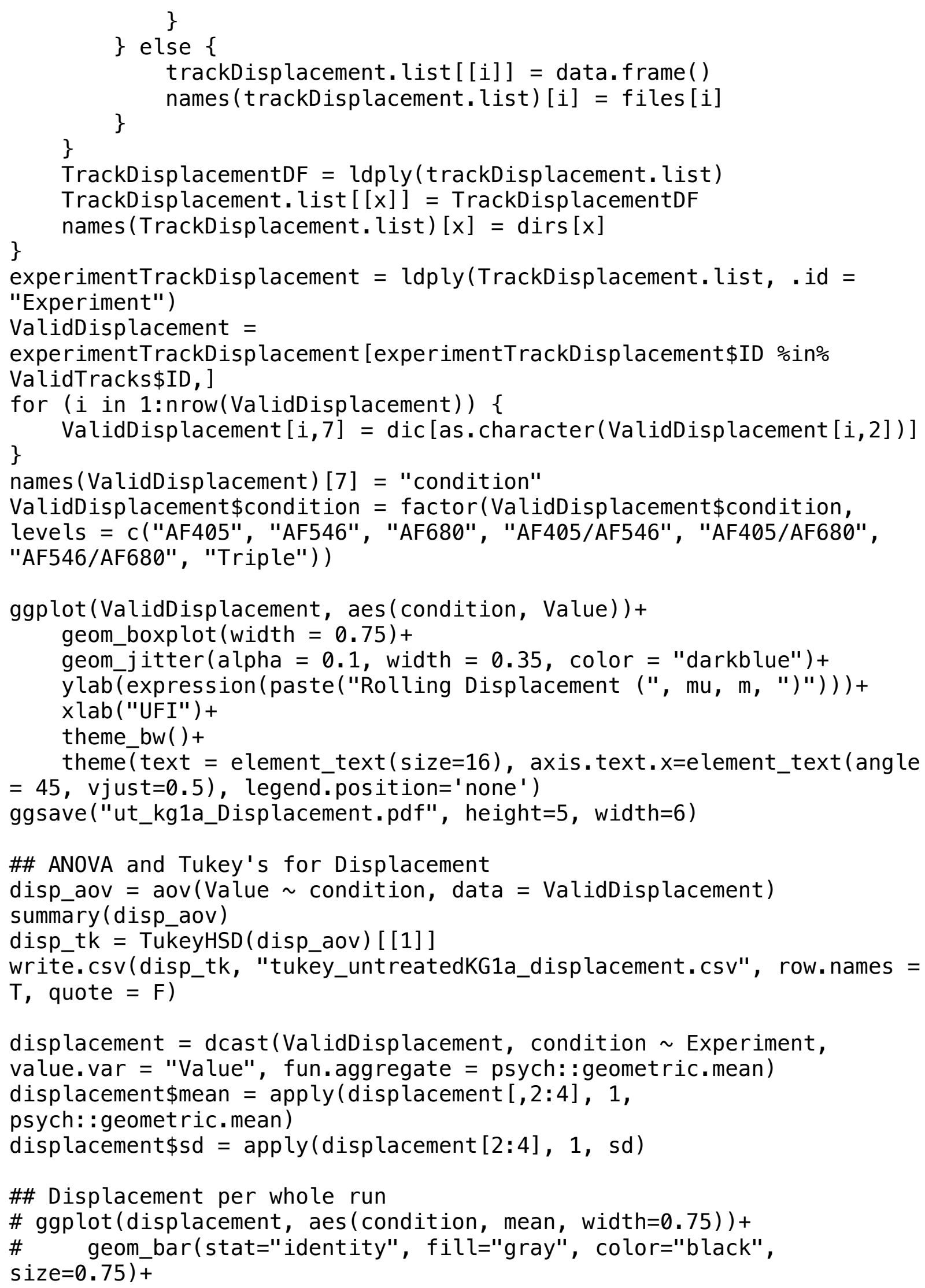




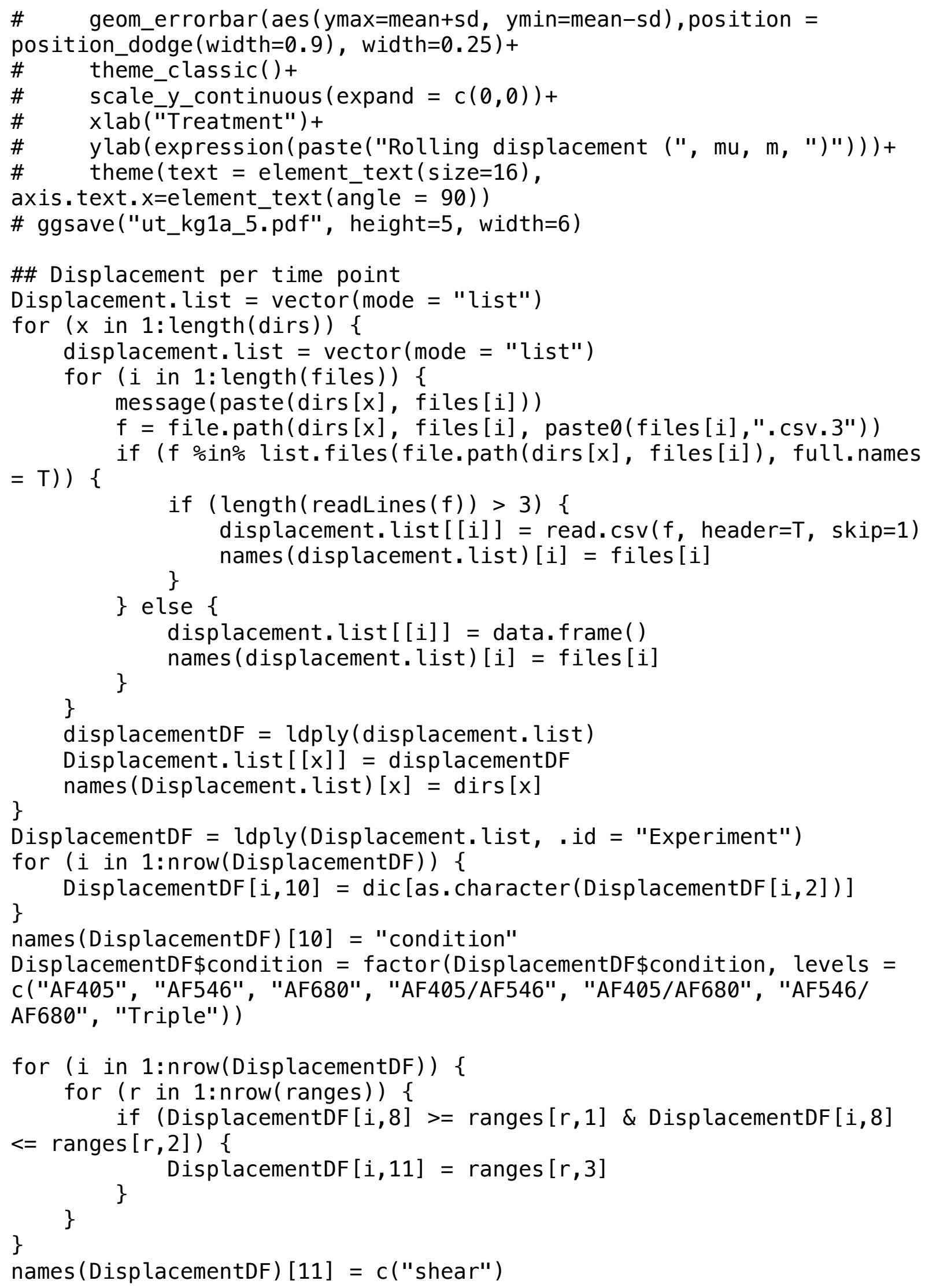


flowDisplacement. list $=$ split (DisplacementDF, DisplacementDF\$Experiment)

mean. flowDisplacement. list $=\operatorname{vector}($ mode $=$ "list" $)$

for ( $i$ in 1: length ( flowDisplacement. list)) \{ mean.flowDisplacement. list [ [i]] =

melt (dcast ( flowDisplacement. list [ [i]] [f lowDisplacement. list [ [i] ] \$Time\% $\% 30==0$,$] , condition \sim$ shear, value.var = "Displacement. $X "$, fill $=0$, fun. aggregate $=$ mean) ) \}

mean. flowDisplacement. DF = cbind (mean. flowDisplacement. list [ [1] ] , mean. f lowDisplacement. list [[2] ]\$value, mean. flowDisplacement. list [ [3] ] \$value)

mean. flowDisplacement.DF\$mean = apply(mean. flowDisplacement.DF [, 3:5], 1 , mean )

mean.flowDisplacement.DF\$sd = apply(mean.flowDisplacement.DF [, 3:5], $1, \mathrm{sd})$

\#mean.flowSpeed.DF\$variable $=$ as. numeric (as. character (mean. flowSpeed. DF\$variable))

\#mean. flowspeed. DF = mean. flowSpeed. DF [as. numeric (as. character (mean. flowSpeed. DF\$variable)) $<20$, ]

as.numeric.factor <- function( $x$ ) \{as.numeric(levels $(x))[x]$ \}

mean. flowDisplacement. DF $\$$ variable $=$

as. numeric. factor (mean. flowDisplacement. DF\$variable)

ggplot(mean. flowDisplacement.DF, aes(variable, -mean,

group=condition) ) + geom_point $($ size $=1.5)+$ geom_line(aes (variable,-mean), size $=0.5)+$

geom_errorbar (aes $(y \max =-$ mean+sd, ymin=-mean-sd), width=0.25)+ theme_bw ( )+

facet_grid(. $\sim$ condition $)+$

theme ( text=element_text $($ size $=16))+$

xlab(expression (paste("Shear stress (dyne/cm"^"2",")")))+

ylab(expression (paste("Displacement (", mu,m,")")))

ggsave("ut_kgla_DisplacementPerShear.pdf", height=3, width=18)

\#\# ANOVA and Tukey's

DisplacementDF. endofcycle $=$ DisplacementDF [DisplacementDF $\$$ Time $\% 30$ $==0$, ]

dic2 = factor (c("2", "5", "7", "10", "15", "20"))

names $($ dic2 $)=c(30,60,90,120,150,180)$

DisplacementDF. endofcycle\$shear =

dic [as . character (DisplacementDF. endofcycle\$Time) ]

\# disShear_aov $=$ aov $($ Displacement. $X \sim$ condition $*$ shear, data $=$ DisplacementDF.endofcycle) 


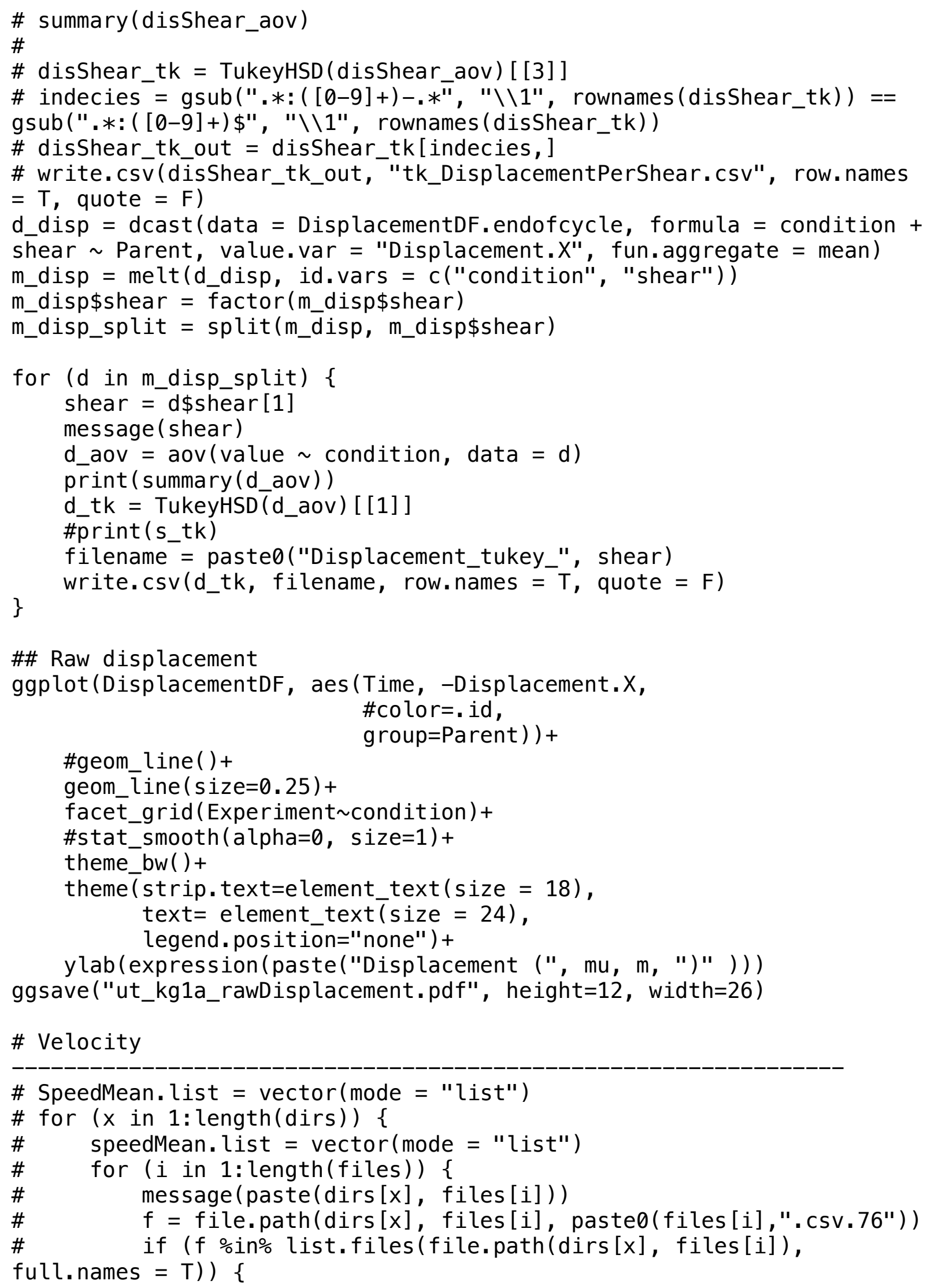




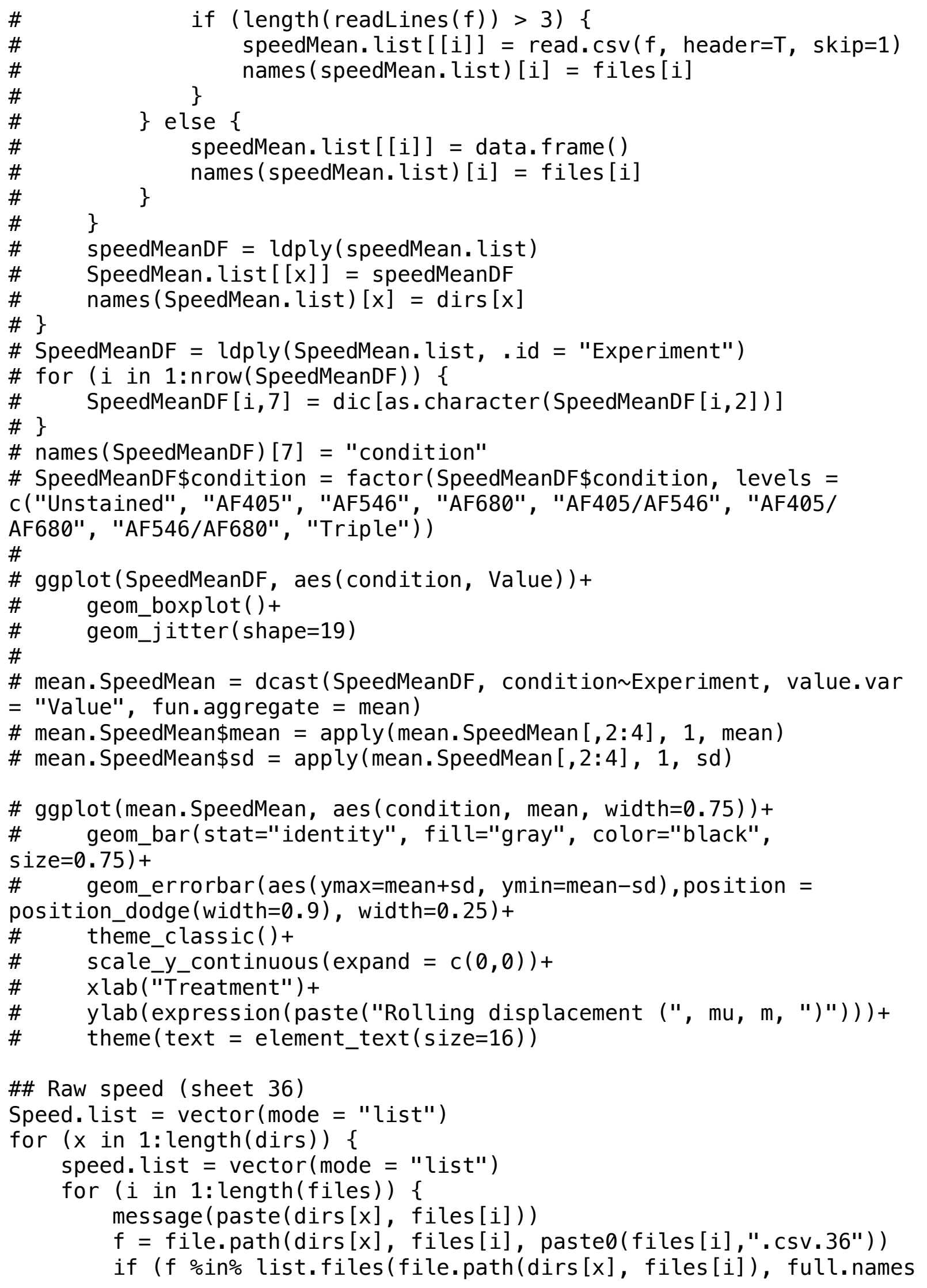




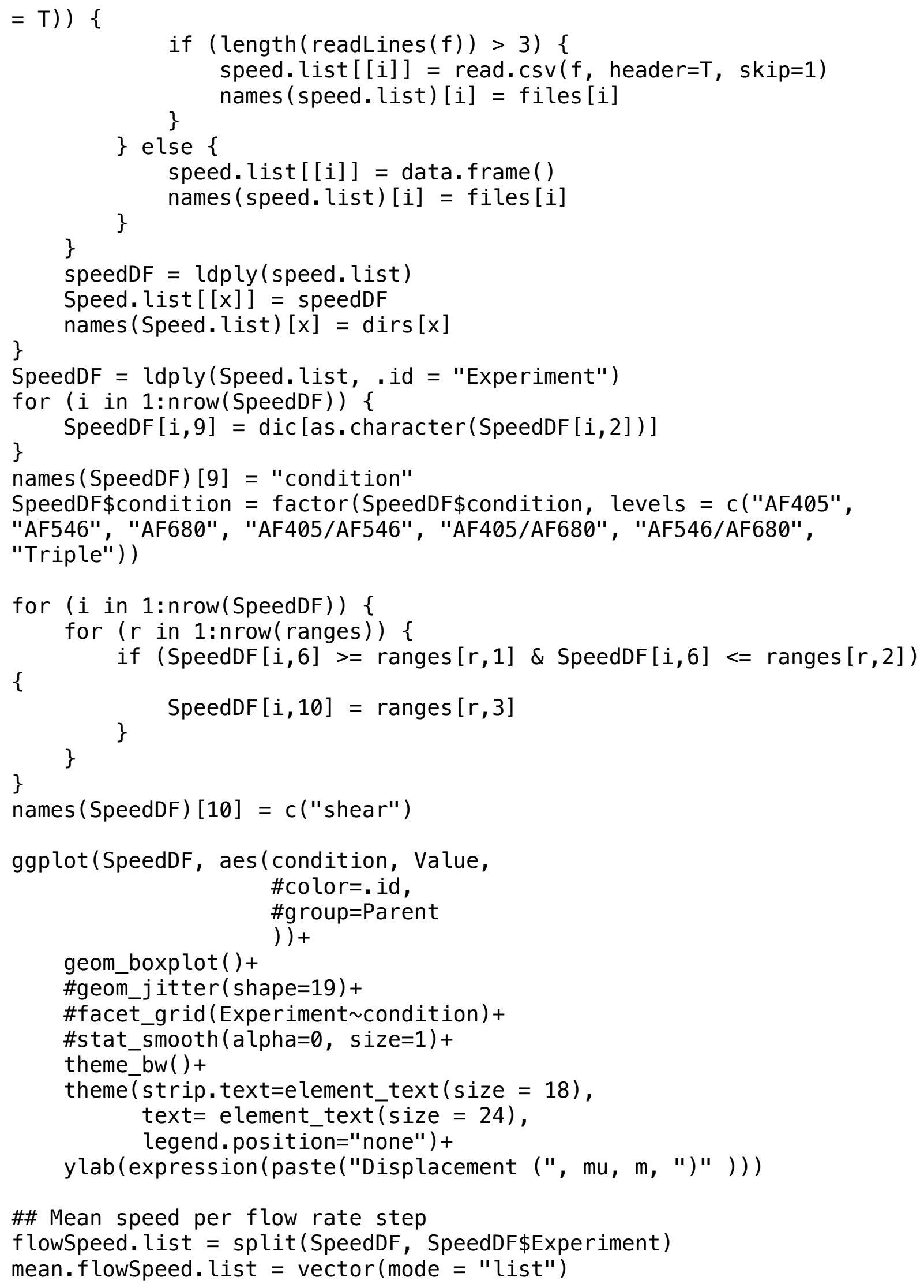




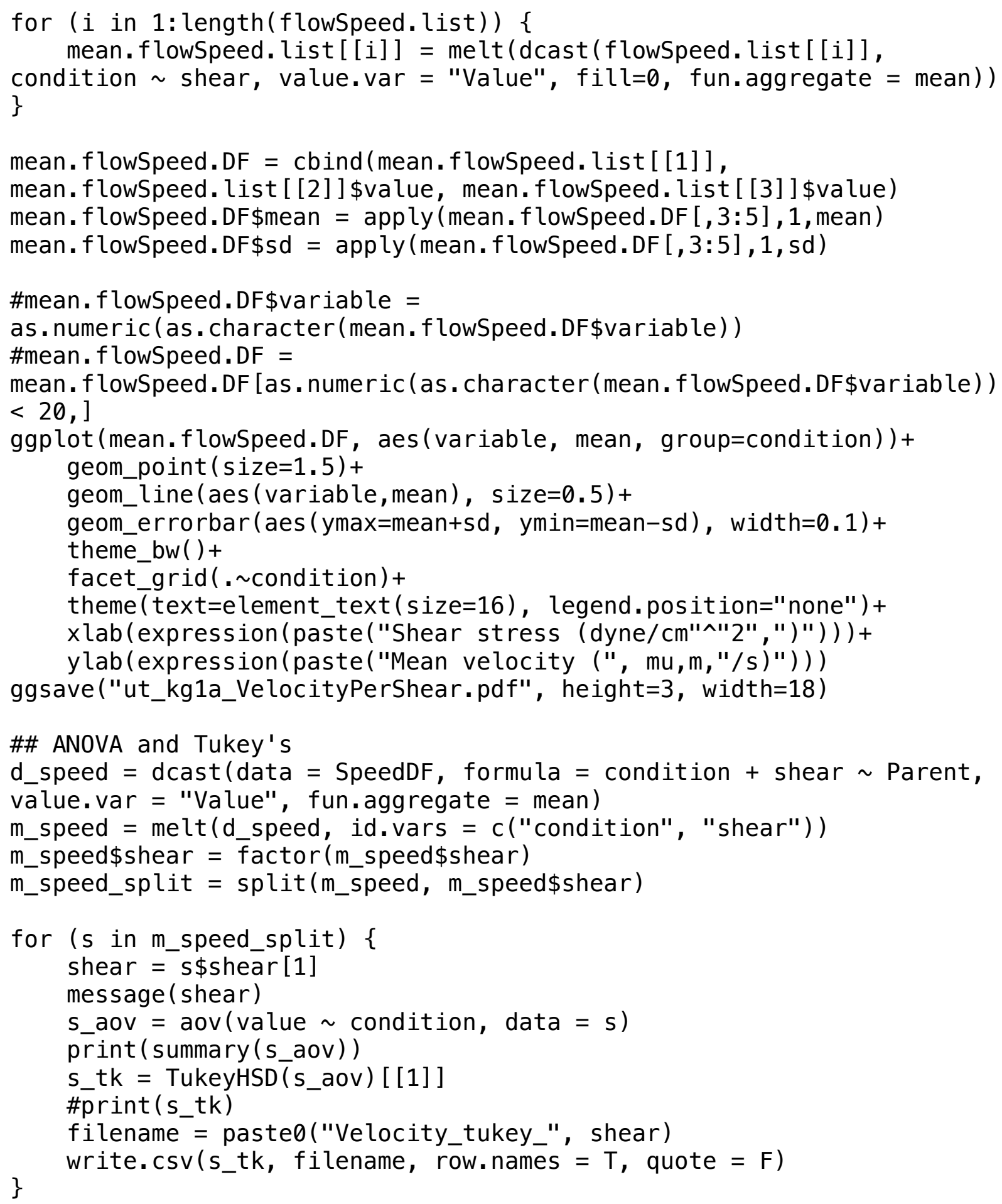




\section{REFERENCES}

1. AbuSamra, D. B.; Al-Kilani, A.; Hamdan, S. M.; Sakashita, K.; Gadhoum, S. Z.; Merzaban, J. S., Quantitative Characterization of E-selectin Interaction with Native CD44 and P-selectin Glycoprotein Ligand-1 (PSGL-1) Using a Real Time Immunoprecipitation-based Binding Assay. J Biol Chem 2015, 290 (35), 21213-30.

2. AbuSamra, D. B.; Aleisa, F. A.; Al-Amoodi, A. S.; Jalal Ahmed, H. M.; Chin, C. J.; Abuelela, A. F.; Bergam, P.; Sougrat, R.; Merzaban, J. S., Not just a marker: CD34 on human hematopoietic stem/progenitor cells dominates vascular selectin binding along with CD44. Blood Adv 2017, 1 (27), 27992816.

3. Aleisa, F. A.; Sakashita, K.; Lee, J. M.; AbuSamra, D. B.; Al Alwan, B.; Nozue, S.; Tehseen, M.; Hamdan, S. M.; Habuchi, S.; Kusakabe, T.; Merzaban, J. S., Functional binding of E-selectin to its ligands is enhanced by structural features beyond its lectin domain. The Journal of biological chemistry 2020, 295 (11), 3719-3733.

4. Sackstein, R.; Merzaban, J. S.; Cain, D. W.; Dagia, N. M.; Spencer, J. A.; Lin, C. P.;

Wohlgemuth, R., Ex vivo glycan engineering of CD44 programs human multipotent mesenchymal stromal cell trafficking to bone. Nat Med 2008, 14 (2), 181-7.

5. Merzaban, J. S.; Imitola, J.; Starossom, S. C.; Zhu, B.; Wang, Y.; Lee, J.; Ali, A. J.; Olah, M.; Abuelela, A. F.; Khoury, S. J.; Sackstein, R., Cell surface glycan engineering of neural stem cells augments neurotropism and improves recovery in a murine model of multiple sclerosis. Glycobiology 2015, 25 (12), $1392-409$. 